\title{
Kinematics of the Ursa Major Molecular Clouds
}

\section{Citation}

Pound, Marc W., and Alyssa A. Goodman. 1997. "Kinematics of the Ursa Major Molecular Clouds." The Astrophysical Journal 482 (1): 334-54. https://doi.org/10.1086/304136.

\section{Permanent link}

http://nrs.harvard.edu/urn-3:HUL.InstRepos:41397496

\section{Terms of Use}

This article was downloaded from Harvard University's DASH repository, and is made available under the terms and conditions applicable to Other Posted Material, as set forth at http:// nrs.harvard.edu/urn-3:HUL.InstRepos:dash.current.terms-of-use\#LAA

\section{Share Your Story}

The Harvard community has made this article openly available.

Please share how this access benefits you. Submit a story.

Accessibility 
The Astrophysical Journal, 482:334-354, 1997 June 10

(C) 1997. The American Astronomical Society. All rights reserved. Printed in U.S.A.

\title{
KINEMATICS OF THE URSA MAJOR MOLECULAR CLOUDS ${ }^{1}$
}

\author{
MARC W. POUND \\ Radio Astronomy Laboratory, University of California, Berkeley, CA 94720; pound@teddi.berkeley.edu \\ AND \\ ALYsSa A. Goodman ${ }^{2}$ \\ Department of Astronomy, Harvard University, Cambridge, MA 02138; agoodman@cfa.harvard.edu \\ Received 1996 October 4; accepted 1997 January 2
}

\begin{abstract}
We present a kinematic analysis of the atomic and molecular gas in the Ursa Major molecular clouds. The analysis is based on a new $\mathrm{CO}$ survey of the complex made with linear resolution of $0.05 \mathrm{pc}$ and existing $\mathrm{H}$ I observations.

The clouds lie in projection on an expanding shell of material known as the North Celestial Pole loop. The molecular structure of the complex is dominated by several long $(>5 \mathrm{pc})$ filaments, some of which are both extremely straight and extremely narrow $(<0.2 \mathrm{pc}$ across). These filaments are enclosed in a sheath of neutral atomic hydrogen that has a kinematic signature distinct from the surrounding atomic gas. The tips of the filaments are regions of enhanced IRAS 12 and $25 \mu \mathrm{m}$ emission. We find an offset of up to $4 \mathrm{~km} \mathrm{~s}^{-1}$ between the centroid velocities of the $\mathrm{CO}$ and $\mathrm{H} \mathrm{I}$, a large-scale velocity gradient in the gas of $\sim 0.3 \mathrm{~km} \mathrm{~s}^{-1} \mathrm{pc}^{-1}$, and a similar large-scale gradient in the $\mathrm{H} \mathrm{I}$ line width. The $\mathrm{CO}$ velocity field follows a trend similar to the $\mathrm{H}$ I but is much less organized. A weak line width gradient in the $\mathrm{CO}$ may also be present.

We present a model in which the clouds lie near the surface of the expanding bubble and, after having interacted with the bubble's wind, are now slowly "sliding" down the bubble toward the Galactic plane. This model adequately accounts for the atomic-molecular velocity offset, the velocity gradients, the line width gradient, and the IRAS colors.

Subject headings: ISM: clouds - ISM: individual (Ursa Major) — ISM: kinematics and dynamics radio lines: ISM
\end{abstract}

\section{INTRODUCTION}

The Ursa Major clouds were among the many high Galactic latitude molecular clouds (HLCs) discovered by Blitz, Magnani, \& Mundy (1984) and cataloged by Magnani, Blitz, \& Mundy (1985; hereafter MBM). In the MBM catalog, the Ursa Major complex appears as clouds 27-30. Like most of the HLCs, the Ursa Major clouds are of moderate size - a few hundred solar masses and about $10 \mathrm{pc}$ in extent-and are not bound by their own self-gravity. The clouds have been the focus of several studies concerned with their $\mathrm{CO}$ and dust emission (de Vries, Heithausen, \& Thaddeus 1987; Boulanger et al. 1990), their fine-scale structure (Falgarone \& Perault 1988; Joncas, Boulanger, \& Dewdney 1992), their magnetic field and neutral atomic hydrogen content (Goodman et al. 1994; Gir, Blitz, \& Magnani 1994; Myers et al. 1995), and their C II emission (Bock et al. 1993).

The clouds lie in a region of relatively low $\mathrm{H}$ I column density $N(\mathrm{H} \mathrm{I}) \lesssim 10^{19} \mathrm{~cm}^{-2}$ (Kuntz \& Danly 1996). Surrounding this region is an enormous loop of material, known as the North Celestial Pole (NCP) loop (Heiles 1984), that is visible in both $\mathrm{H} I$ and infrared cirrus emission. Figure 1 shows the Infrared Astronomical Satellite (IRAS) $100 \mu \mathrm{m}$ image of the NCP loop. The NCP loop is about 60 pc in diameter and the Ursa Major clouds appear as "fingers" at $(\ell, b) \sim(140,38)$ that "hang down" toward the center of the loop and the plane of the Galaxy. Meyerdierks,

\footnotetext{
${ }^{1}$ This paper is dedicated to Dr. James Jay Klavetter.

${ }^{2}$ National Science Foundation Young Investigator.
}

Heithausen, \& Reif (1991) studied the morphology and kinematics of the NCP loop and concluded it was a highly elongated ellipsoidal shell, with its short axis perpendicular to our line of sight, expanding with a velocity of $10-20 \mathrm{~km}$ $\mathrm{s}^{-1}$. The NCP loop is near, but not coincident with, the so-called Lockman hole, the region of lowest $\mathrm{H}$ I column density in the sky (Lockman, Jahoda, \& McCammon 1986; Jahoda, Lockman, \& McCammon 1990; Heiles \& Habing 1974).

Penprase (1993) observed interstellar absorption lines toward stars projected on the clouds and determined the distance to MBM 30 to be $d=110 \pm 10 \mathrm{pc}$ and an upper limit on the distance to MBM 29 of $346 \mathrm{pc}$. Throughout this paper, we will assume that the distance to all of the clouds is $d=110 \pm 10 \mathrm{pc}$. None of our conclusions are substantially affected by the choice of distance.

Despite all this interest in the Ursa Major region, there has yet to be a detailed study of the kinematics of its molecular clouds. In this paper, we present a kinematical analysis of the clouds, making use of new $\mathrm{CO}$ observations as well as existing $\mathrm{H}$ I and IRAS maps. We propose a model in which the clouds are located on the far side of an expanding bubble associated with the NCP loop. The model accounts not only for the gas velocity and line width gradients but also for the difference in centroid velocity between the atomic and molecular gas and for large-scale variations across the clouds in the IRAS colors.

After describing our observations in $\S 2$, we present in $\S 3$ the molecular, atomic, and dust emission maps, as well as maps of derived quantities such as centroid velocity, line 


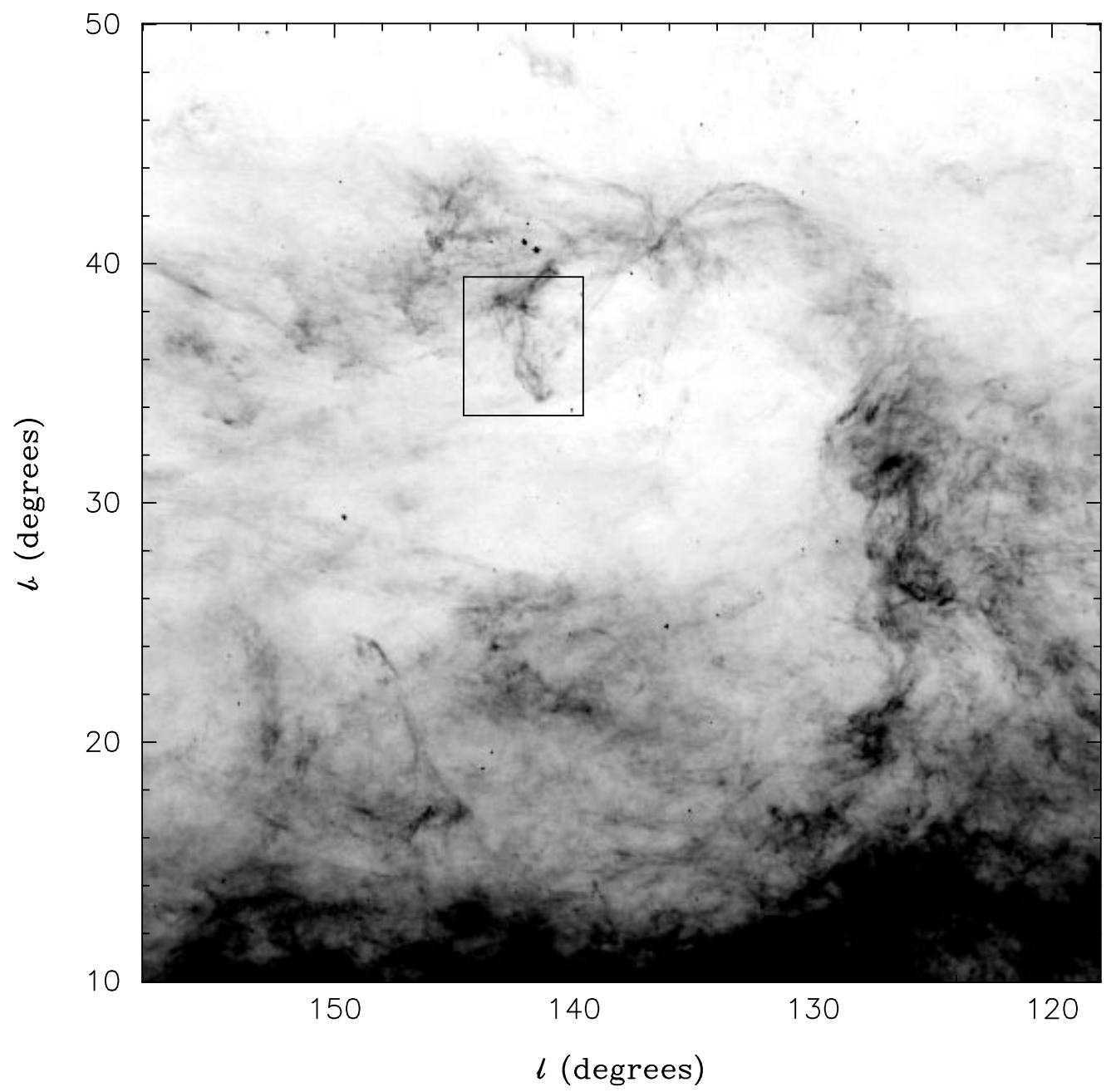

Fig. 1.-IRAS $100 \mu \mathrm{m}$ flux density image of the North Celestial Pole loop. The Ursa Major molecular clouds are the features that "hang down" from the top of the loop at $(\ell, b) \sim(140,38)$. The area covered by the $\mathrm{H}$ I and CO maps is indicated with a box.

width, and color temperature, and we point out salient features of the clouds. Our model for the Ursa Major complex is described in $\S 4$, wherein we also test the model predictions against the observations. Our conclusions are summarized in $\S 5$.

\section{OBSERVATIONS AND DATA REDUCTIONS ${ }^{3}$}

\subsection{Carbon Monoxide}

We used the AT\&T Bell Laboratories $7 \mathrm{~m}$ telescope located in Holmdel, NJ to map the Ursa Major clouds (MBM 27-30) in the CO(1-0) transition at $115.271203 \mathrm{GHz}$ during the 1988-1990 observing seasons. At $115 \mathrm{GHz}$, the 7 $\mathrm{m}$ antenna has a symmetric Gaussian beam $100^{\prime \prime}$ in diameter (HPBW). The spectra were taken by in-band frequency switching, with signal and reference frequencies offset by 6.4

${ }^{3} \mathrm{We}$ have constructed three-dimensional representations of the $\mathrm{CO}$ and $\mathrm{H}$ I data presented herein using Virtual Reality Markup Language (VRML). These representations can be viewed on the World Wide Web in the NCSA Digital Image Library at http://imagelib.ncsa.uiuc.edu/document/96.MP.01 using any browser that supports VRML.
MHz. The IF signals were sent to a multichannel filterbank, which consists of 256 channels with a resolution of $250 \mathrm{kHz}$ per channel. The signals from one-half of the channels were sent to a spectrum expander to give a resolution of $100 \mathrm{kHz}$ or $0.27 \mathrm{~km} \mathrm{~s}^{-1}$ at the observing frequency. Total system temperatures including sky noise were between 200 and $1000 \mathrm{~K}$. Line intensities were converted to corrected antenna temperatures, $T_{R}^{*}$, by applying corrections for atmospheric attenuation and telescope efficiency $(\eta=90 \%)$. Linear or second-order baselines were removed from all spectra. The typical rms noise per spectrum was $0.5-1 \mathrm{~K}$. In all, 21,424 spectra spaced on $1^{\prime}$ grid and covering about 5 $\mathrm{deg}^{2}$ were obtained. The spectra were taken on an equatorial coordinate system grid; however, for comparison with existing $\mathrm{H}$ I maps, we computed the $\mathrm{CO}$ maps in Galactic coordinates.

When CO spectra are taken in frequency-switched mode, the telluric CO line can appear in the observing band at $-V_{\text {ant }}$, where $V_{\text {ant }}$ is the velocity of the antenna with respect to the local standard of rest (LSR). Alternatively, the image of the telluric CO line can appear as absorption somewhere in the band, the exact location depending on the separation between the signal and reference frequencies. The telluric 
CO line can therefore contaminate the map, and care must be taken to remove it during data processing. We determined the strength and LSR velocity of the telluric line each day by observing an empty patch of sky. Using the sky spectra as guides, we "blanked out" channels in our spectra in which the telluric line appears. In the few cases in which the telluric line was too close in velocity to the spectral line from the molecular cloud, we discarded the spectrum. The portions of spectra which had telluric $\mathrm{CO}$ emission were not used in determination of the baselines.

\subsection{Atomic Hydrogen}

$\mathrm{H}$ I observations at $1420.405752 \mathrm{MHz}$ were made in 1990 , 1991, and 1992 with the $26 \mathrm{~m}$ telescope at Hat Creek Radio Observatory as part of a program to measure the Zeeman effect in diffuse clouds. The telescope had angular resolution of 36' (HPBW). Further observations were made in 1991 October and 1992 November with the $100 \mathrm{~m}$ telescope $\left(9^{\prime}\right.$ HPBW) of the Max-Planck-Institut für Radioastronomie in Effelsberg, Germany. The Effelsberg data were spaced on a $15^{\prime}$ grid and cover about $15 \mathrm{deg}^{2}$. Full details of the observation and reduction procedures for both sets of data are given in Myers et al. (1995). In our analysis below, we will use primarily the Effelsberg data, since they have higher resolution and sensitivity than the Hat Creek observations. However, the Zeeman data discussed here were all obtained at Hat Creek.

\subsection{Dust}

To examine far-infrared emission from dust associated with the Ursa Major clouds, we obtained the co-added images of flux density from the IRAS Sky Survey Atlas using the NASA Skyview Image Server. Each image has pixel size of $90^{\prime \prime} \times 90^{\prime \prime}$ and is $19^{\circ} .2$ on a side (768 pixels) with the coordinate of pixel $(250,250)$ at $(\ell, b)=(142.0,37.0)$. The maps have units of $\mathrm{MJy} \mathrm{Sr}^{-1}$, are made with gnomonic projection, and have spatial resolution smoothed to the IRAS resolution at $100 \mu \mathrm{m}$ (approximately $5^{\prime}$ ). Background subtraction was accomplished as follows. We found three regions of apparently empty sky by inspection of the 100 $\mu \mathrm{m}$ image. For each wave band, the mean flux density in these regions was determined and the plane of mean flux density passing through the midpoints of the three regions (points $\mathrm{P}, \mathrm{Q}$, and $\mathrm{R}$ in Table 1) was fitted and subtracted.
The same regions/midpoints were used for all four IRAS bands. The resulting images had in the chosen regions zero mean flux density and residual noise well characterized by thermal noise. The parameters of the fits are given in Table 1. Once we had satisfactory background-subtracted maps, we extracted subimages of similar extent as our $\mathrm{CO}$ and $\mathrm{H} \mathrm{I}$ maps. Figure 2 shows the background-subtracted IRAS flux density at $100 \mu \mathrm{m}, I(100)$.

Dust temperature and opacity maps were computed from the IRAS images using the method described in Langer, Wilson, \& Beichman (1989) and computer subroutines provided by IPAC. Briefly, the method is as follows. Assuming the dust in a single beam can be characterized by one temperature $T_{d}$, the ratio of flux densities $F$ at two wavelengths $\lambda_{i}$ and $\lambda_{j}$ is given by

$$
R \equiv \frac{F_{i}}{F_{j}}=\frac{B\left(T_{d}, \lambda_{i}\right) \tau_{i}}{B\left(T_{d}, \lambda_{j}\right) \tau_{j}} \frac{\Omega_{i}}{\Omega_{j}}
$$

where $B(T, \lambda)$ is Planck function, $\tau$ is the optical depth, and $\Omega$ is the beam solid angle. If it is assumed that the emission is optically thin, that the optical depth follows a power law with wavelength $\tau \propto \lambda^{-a}$, and that $\Omega_{i}=\Omega_{j}$, then the expression for $R$ becomes

$$
R=\left(\frac{\lambda_{j}}{\lambda_{i}}\right)^{a-3}\left[\frac{\exp \left(T_{i} / T_{d}\right)-1}{\exp \left(T_{j} / T_{d}\right)-1}\right]
$$

where $T_{i}=h c / \lambda_{i} k$. Starting with the ratio map of the IRAS flux density at two adjacent wave bands, equation (2) is solved for $T_{d}$ using linear interpolation of a lookup table of $R$ (before color correction - see below). The resulting temperatures are clipped at about 20 and $10,000 \mathrm{~K}$; the exact clipping value depends on the wavelengths of the input images.

Before calculating the opacity map at any wavelength, a color correction must be applied to the flux density map to account for the effects of the finite IRAS passbands. The color correction is performed as described by Wheelock et al. (1991). Dividing each pixel of a color-corrected flux density map by the Planck function at the derived temperature of that pixel converts the flux map into an opacity map at that wavelength. The input flux density maps were each clipped at twice their rms noise levels indicated in Table 1. We assumed $a=1$ (Hildebrand 1983). Figure 3 is the $\tau_{100}$ maps, and Figure 4 is the $T(12 / 25)$ map. The fila-

TABLE 1

\begin{tabular}{|c|c|c|c|c|c|c|c|}
\hline \multirow{2}{*}{$\begin{array}{l}\text { BAND } \\
(\mu \mathrm{m})\end{array}$} & \multirow{2}{*}{$\begin{array}{c}I(\mathrm{P})^{\mathrm{a}} \\
\left(\mathrm{MJy} \mathrm{Sr}^{-1}\right)\end{array}$} & \multirow{2}{*}{$\begin{array}{c}I(\mathrm{Q})^{\mathrm{b}} \\
\left(\mathrm{MJy} \mathrm{Sr}^{-1}\right)\end{array}$} & \multirow{2}{*}{$\begin{array}{c}I(\mathrm{R})^{\mathrm{c}} \\
\left(\mathrm{MJy} \mathrm{Sr}^{-1}\right)\end{array}$} & \multicolumn{3}{|c|}{ PLANE $^{d}$} & \multirow{2}{*}{$\begin{array}{c}\text { ResiduAL RMS } \\
\left(\mathrm{MJy} \mathrm{Sr}^{-1}\right)\end{array}$} \\
\hline & & & & A & B & $\mathrm{C}$ & \\
\hline $100 \ldots \ldots$ & 0.044 & -0.015 & 0.033 & $8.27 \times 10^{-5}$ & $-1.85 \times 10^{-5}$ & $2.90 \times 10^{-3}$ & 0.140 \\
\hline $60 \ldots \ldots$ & -0.690 & -0.995 & -0.780 & $4.35 \times 10^{-4}$ & $-1.30 \times 10^{-5}$ & -0.997 & 0.065 \\
\hline $25 \ldots \ldots$ & 4.690 & 4.130 & 4.480 & $7.99 \times 10^{-4}$ & $4.39 \times 10^{-5}$ & 4.077 & 0.064 \\
\hline $12 \ldots \ldots$ & 1.130 & 0.990 & 1.160 & $2.06 \times 10^{-4}$ & $-1.58 \times 10^{-4}$ & 1.097 & 0.070 \\
\hline
\end{tabular}

Planes Fitted to IRAS BACKGRoUnd

${ }^{\text {a }}$ Intensity at pixel $P=(726.5,739)$ given as the mean intensity in a box with bottom left-hand corner at pixel $(686,711)$ and top right-hand corner at pixel $(767,767)$.

${ }^{\mathrm{b}}$ Intensity at pixel $\mathrm{Q}=(25.5,742.5)$ given as the mean intensity in a box with bottom left-hand corner at pixel $(1,717)$ and top right-hand corner at pixel $(50,768)$.

${ }^{c}$ Intensity at pixel $\mathrm{R}=(491,242.5)$ given as the mean intensity in a box with bottom left-hand corner at pixel $(472,220)$ and top right-hand corner at pixel $(510,285)$.

${ }_{\mathrm{d}}$ Plane of the form $I\left(\mathrm{MJy} \mathrm{Sr}^{-1}\right)=\mathrm{A} x+\mathrm{B} y+\mathrm{C}$ fitted to intensities at points $\mathrm{P}, \mathrm{Q}$, and $\mathrm{R}$ and subtracted from raw image.

${ }^{e}$ rms noise in emission-free regions of image after subtraction of background plane. 


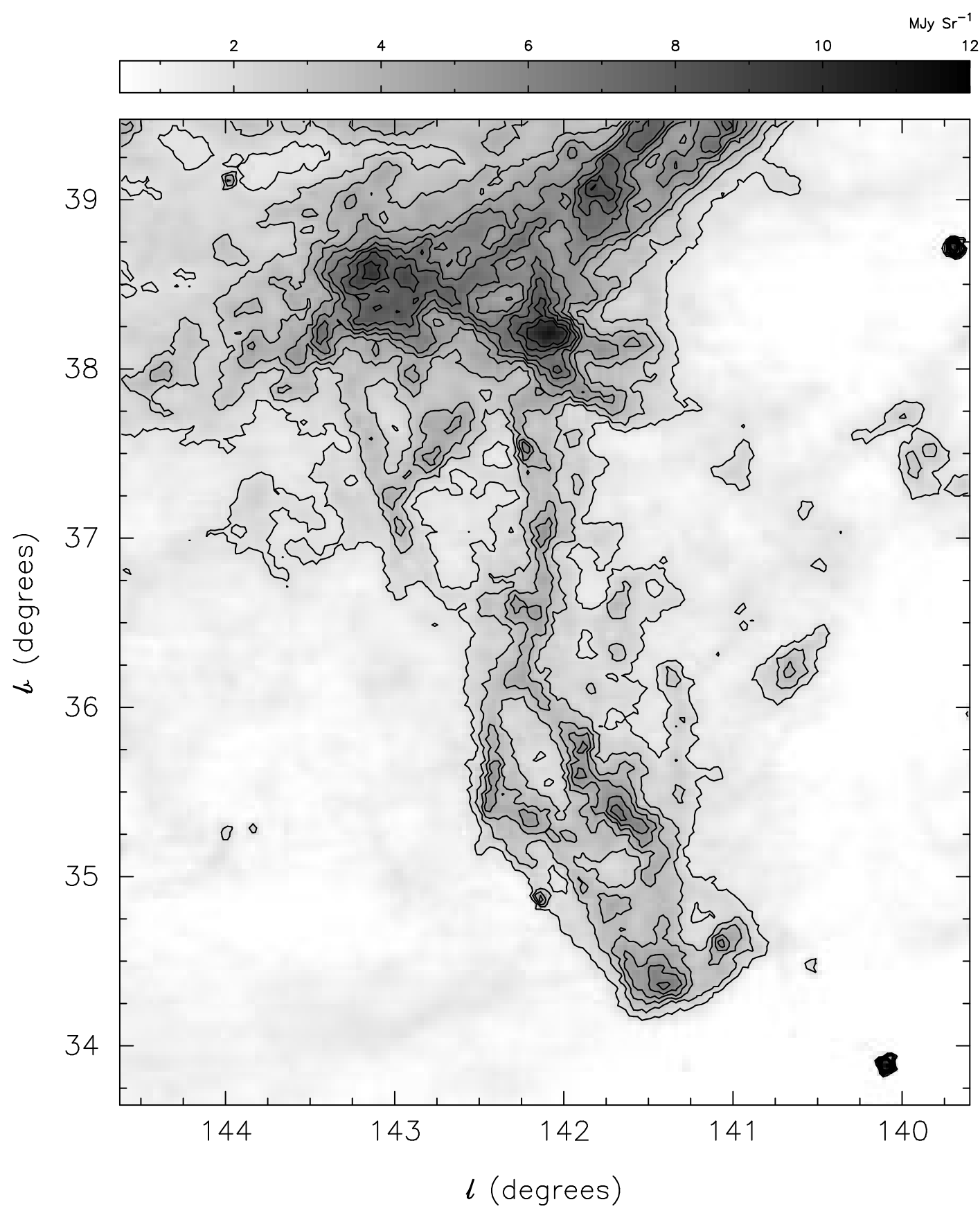

FIG. 2.-Background-subtracted IRAS $100 \mu \mathrm{m}$ flux density image of the Ursa Major clouds. Halftone scale is $0.45-12 \mathrm{MJy} \mathrm{Sr}^{-1}$. Contours (6 $\sigma$ intervals starting at $12 \sigma$ ) are at $0.18,0.27, \ldots, 9.0 \mathrm{MJy} \mathrm{Sr}^{-1}$.

mentary features in the $I(100)$ and $\tau_{100}$ maps correlate in detail with similar features in the CO map.

\section{RESULTS}

\subsection{Morphological Features}

The large-scale IRAS image in Figure 1 offers a contextual view of the Ursa Major clouds. In projection, the NCP loop appears as a ring of $100 \mu \mathrm{m}$ emission, with the Ursa Major clouds near the top of the ring. The Ursa Major filaments are readily apparent, and they appear, in projection, to be about $17 \%$ of the ring diameter in length. Closer inspection of the IRAS image reveals a wealth of puzzling complexity. Long arcing linear features can be seen crossing the filaments, and sub-bubble-like structures can also be discerned. Because all of these features are seen projected on top of each other, it is hard to tell which features are more or less directly related to which others. In this paper, we will rely on spectral line information, which offers a third dimension (velocity), in our attempts to disentangle various features.

Figure 5 shows the $\mathrm{CO}$ integrated intensity, $I\left({ }^{12} \mathrm{CO}\right)$, in Ursa Major over all velocities present in the molecular gas, from $v_{\text {LSR }}=-2.0$ to $3.5 \mathrm{~km} \mathrm{~s}^{-1}$. The cloud morphology is dominated by two parallel filaments some $8 \mathrm{pc}$ long in projection, which correspond to similar features in the IRAS maps (marked as filaments 1 and 2 in Fig. 6; see also Fig. 8). The filaments connect up (or twist) at the midregion of the 


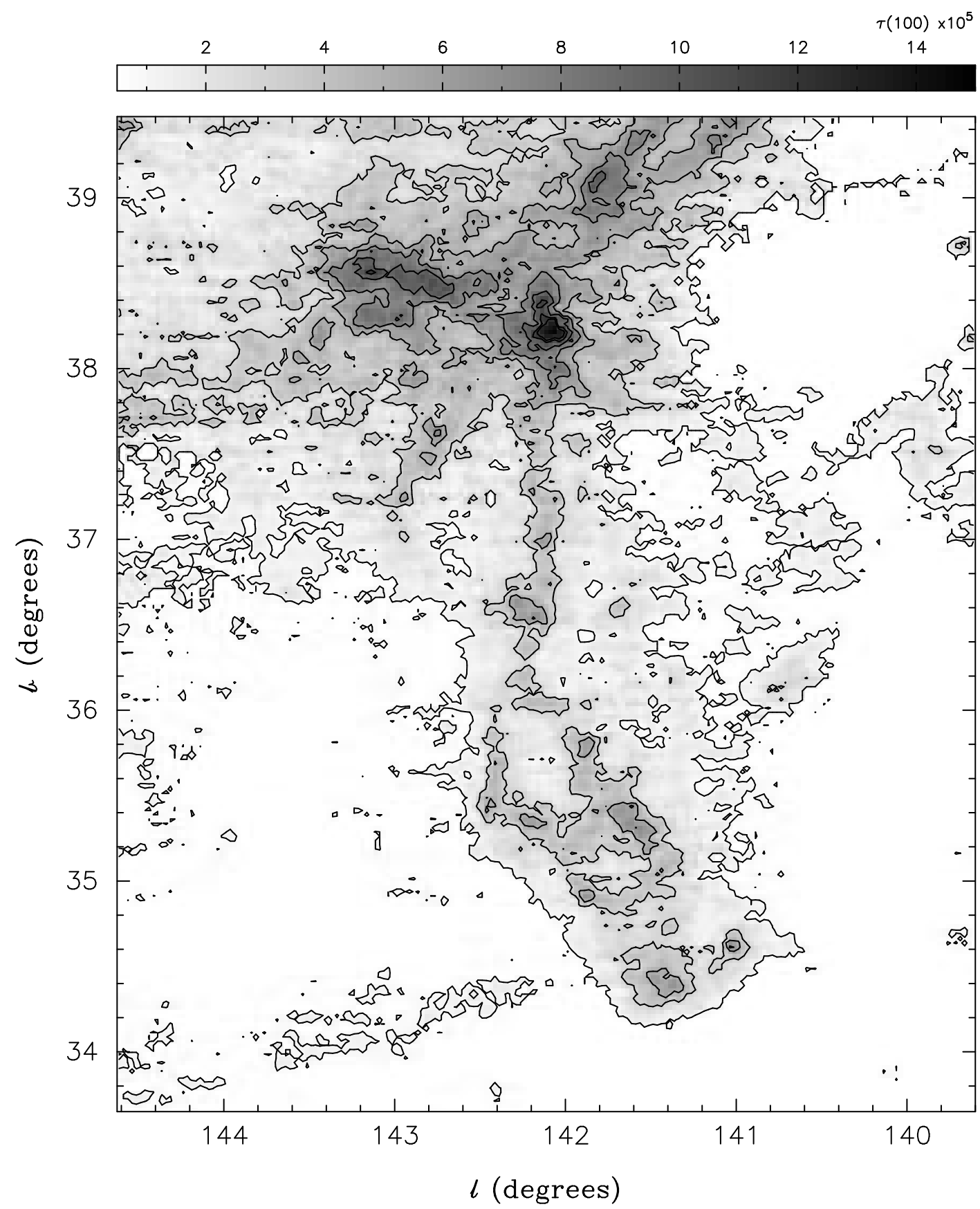

FIG. 3. $-I R A S 100 \mu \mathrm{m}$ opacity calculated from the $T(60 / 100)$ and $100 \mu \mathrm{m}$ images. A $\lambda^{-1}$ opacity law was assumed. Contours are at $2 \times 10^{-6}, 4 \times$ $10^{-6}, \ldots, 12 \times 10^{-6}$. The $100 \mu$ m opacity correlates well with CO integrated intensity in Fig. 5.

complex, and then at the higher latitudes, they merge with the molecular gas that is parallel to - and presumed to be associated with - the $\mathrm{H}$ I shell (MBM 30). In the CO channel maps (Fig. 7), the clouds appear very "clumpy," and condensations ranging in size from a few tenths of a parsec all the way down to the beam size $(0.054 \mathrm{pc})$ can be identified.

The detailed filamentary structure of the $\mathrm{CO}$ clouds is also worthy of investigation. There are a number of extremely long "subfilaments," which are both very narrow (aspect ratios of about 15) and very straight. Some examples of these structures are shown in Figure 8. Corresponding structures are visible in the IRAS 100 and $60 \mu \mathrm{m}$ maps, although the poorer resolution of the IRAS data and an unresolved $100 \mu \mathrm{m}$ source in Figure $8 a$ makes discerning such fine features difficult. The subfilament widths are only marginally resolved at the resolution of our $\mathrm{CO}$ observations, yet they extend for over a parsec. Even more remarkably, the two shown in Figure $8 a$ are parallel to within $1^{\circ}$ for their entire length. In the other subfilament (Fig. 8b), the clumps appear as "beads on a string" with the "beads" separated by about 0.3 pc. We also note that the tips of filaments 1 and 2 appear as a flattened arc when viewed over a selected velocity range, as if they were being compressed and swept back (see Fig. 9 and the panels covering $v_{\mathrm{LSR}}=0-1 \mathrm{~km} \mathrm{~s}^{-1}$ of Fig. 7). 


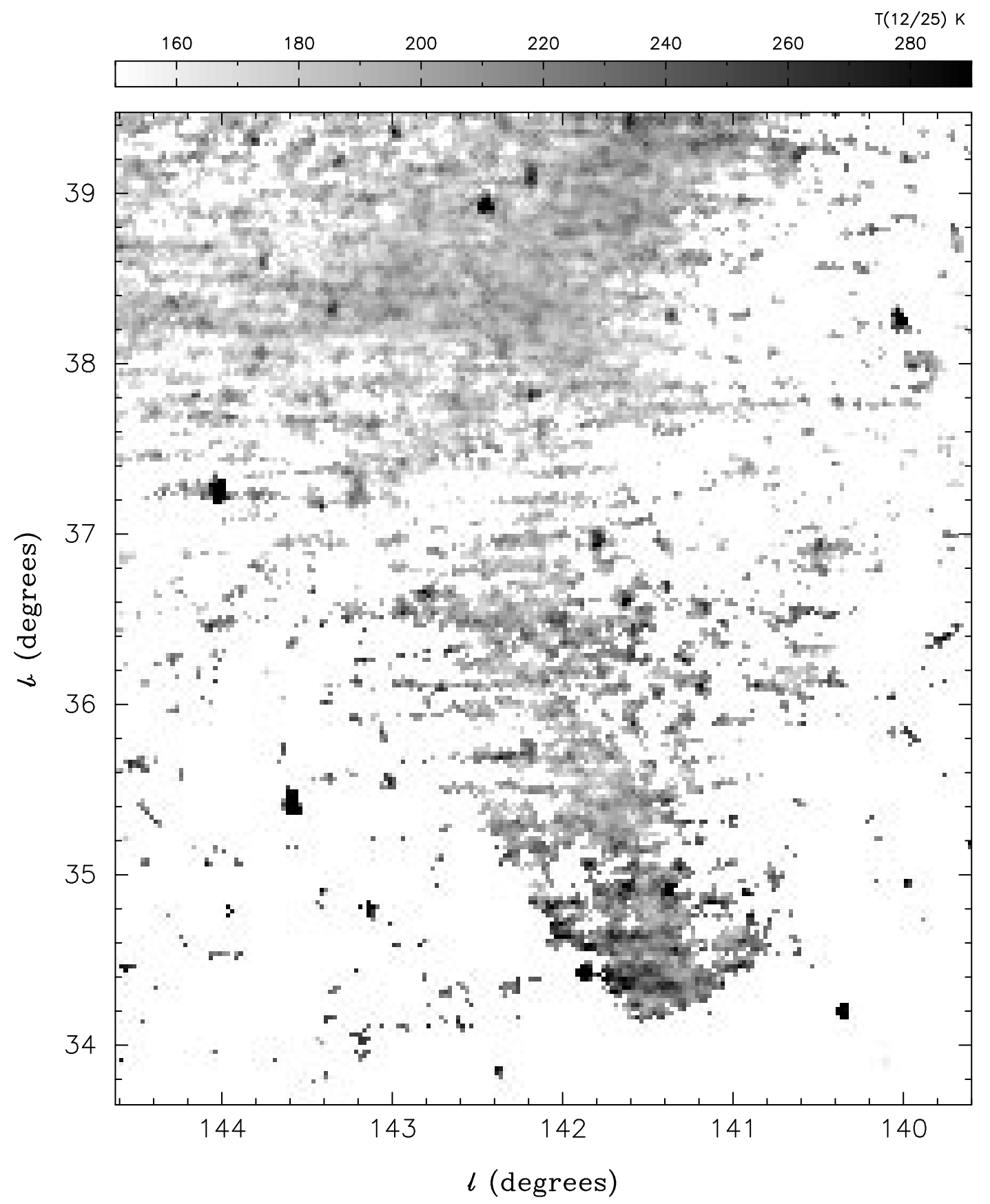

FIG. 4. IRAS T(12/25) dust temperature calculated from the background-subtracted $25 \mu \mathrm{m}$ and $12 \mu \mathrm{m}$ images. The increased dust temperature near the bottom of the filaments (MBM 27) suggest a recent heating event, such as a shock.

\subsection{Temperature Structure}

The tips of filaments 1 and 2 , between roughly $142.4 \gtrsim$ $\ell \gtrsim 140.6$ and $35 \gtrsim b \gtrsim 34$, are regions of enhanced dust temperature $T(12 / 25)$ and color ratio $R(12,25)=I(12) / I(25)$ (see Fig. 4). Inside this region, the value of these quantities are $T(12 / 25)=221 \pm 2.4 \mathrm{~K}$ and $R(12 / 25)=0.84 \pm 0.02$, while outside the region they are $T(12 / 25)=192 \pm 1 \mathrm{~K}$ and $R(12 / 25)=0.57 \pm 0.01$. These dramatic variations in the IRAS temperature colors may indicate an enhancement in the abundance of small particles at the tips of the filaments (see $\S 4.2 .4)$.

\subsection{Velocity Structure}

The centroid velocities of the molecular clumps in the complex fall into three ranges, roughly $v_{\mathrm{LSR}}=-1$ to $0 \mathrm{~km}$ $\mathrm{s}^{-1}, 0-1 \mathrm{~km} \mathrm{~s}^{-1}$, and 1-2 $\mathrm{km} \mathrm{s}^{-1}$. A color map of centroid velocity, $v_{\text {cent }}(\mathrm{CO})$, is shown in Figure 10 (Plate 8). Filaments 1 and 2 share the same kinematics, with a gradient of about $-0.7 \mathrm{~km} \mathrm{~s}^{-1} \mathrm{pc}^{-1}$ along their length. Plots of $v_{\text {cent }}(\mathrm{CO})$ and FWHM line width, $\Delta v$, versus Galactic latitude are shown in Figure 11. A velocity gradient with respect to $b$ can be seen in the $\mathrm{CO}$, but the velocity field is clearly complex. From the figure, there does not appear to be any systematic gradient in FWHM line width, although a weak trend may be present (see $\S 3.3 .2$ ).

Figure 12 shows the $\mathrm{H}_{\mathrm{I}}$ integrated intensity map and a few sample spectra from the Effelsberg data. The atomic hydrogen appears as a single filament that forms a sheath around the $\mathrm{CO}$ filaments that connects to the NCP loop at 


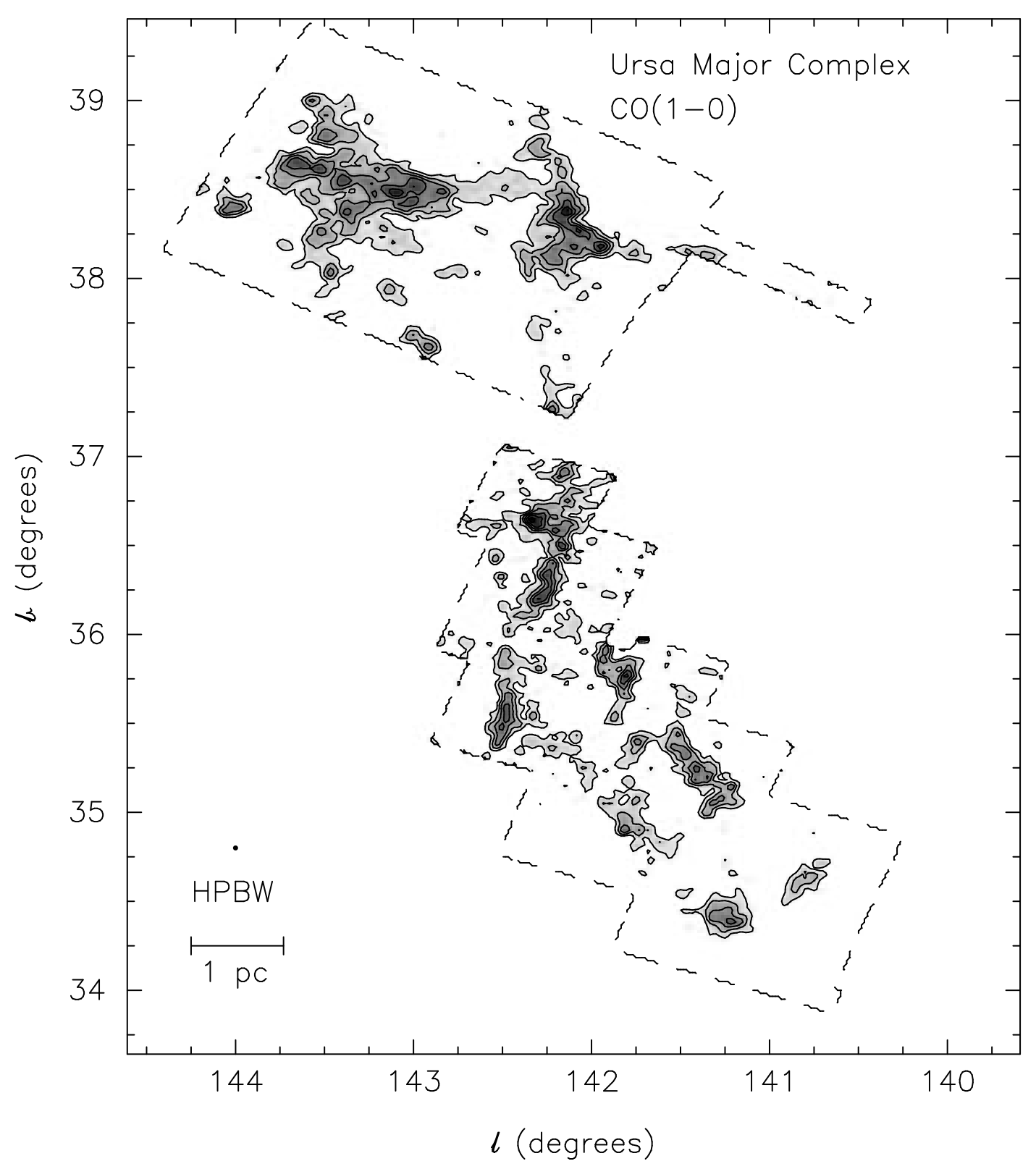

FIG. 5.-Position-position contour map of the integrated intensity of the $\mathrm{CO}(1-0)$ emission between -2 and $3.5 \mathrm{~km} \mathrm{~s}{ }^{-1}$ LSR in the Ursa Major molecular cloud complex. Spectra are spaced on a 1' grid inside the boxed region. The data have been smoothed with a conical filter with interpolation radius $2^{\prime}$. Contours $(2.5 \sigma)$ of the total integrated intensity are drawn at $-1.4,1.4, \ldots, 8.4 \mathrm{~K} \mathrm{~km} \mathrm{~s}^{-1}$. The gray scale range is $0.83-8.9 \mathrm{~K} \mathrm{~km} \mathrm{~s}^{-1}$. The total molecular mass of the complex is about $220 M_{\odot}$.

$b \sim 38^{\circ}$. The gas in the $\mathrm{H}$ I filament has a single velocity component at $v_{\text {LSR }} \sim 2.5 \mathrm{~km} \mathrm{~s}^{-1}$, and the core of $\mathrm{H} \mathrm{I} \mathrm{spec-}$ tral lines there are well approximated by Gaussian profiles. Near the intersection of the filament with the NCP loop (i.e., near $b=37.5$ ), a second velocity component at $v_{\text {LSR }} \sim$ $9 \mathrm{~km} \mathrm{~s}^{-1}$ appears, and the velocity component associated with the filament seems to shift a few $\mathrm{km} \mathrm{s}^{-1}$ blueward. Above $\ell \sim 38^{\circ}$, there are several velocity components present, and the blending of all the lines makes them hard to decompose.

Figure 13 (Plate 9) is a map of $\mathrm{H}_{\text {I }}$ centroid velocity overlaid with contours of $\mathrm{H}$ I peak brightness temperature. The velocity field in the $\mathrm{H}$ I filament is well behaved; as noted previously, a single velocity component is present along with a fairly smooth velocity gradient that is primarily in Galactic latitude. From this figure, we can see what portions of the $\mathrm{H}$ I emission are not directly associated with the atomic filament and therefore are not associated with the molecular clouds. (Note, however, the color scale differs in Figs. 10 and 13.) The regions with extreme values of $v_{\text {cent }}(\mathrm{H} \mathrm{I})\left(\gtrsim 4 \mathrm{~km} \mathrm{~s}^{-1}\right.$; shown as yellow, orange, red, and white in the figure) are well separated in both space and velocity from the sheath. The dramatic change in $v_{\text {cent }}(\mathrm{H} \mathrm{I})$ at the top of the map (the NCP loop/MBM 30 region) is not due to a large shift in the velocity component of the $\mathrm{H} \mathrm{I}$ filament but rather to the additional spectral components mentioned previously. Interestingly, a slight deviation is seen in the smooth behavior of the $\mathrm{H}$ I filament velocity field at the position of the greatest deviation in the $\mathrm{CO}$ velocity field, i.e., in the "blue" regions of filaments 1 and 2, near MBM 28 (see Fig. 10).

In the atomic gas, in addition to the large-scale velocity gradient, we also see a gradient in the FWHM line width (Fig. 11). As with the velocity gradient, the line width gra- 
TABLE 2

Fitted Velocity Gradients to H i and CO Data

\begin{tabular}{lcccc}
\hline \hline \multicolumn{1}{c}{ Map } & $\begin{array}{c}\text { Magnitude } \\
\left(\mathrm{km} \mathrm{s}^{-1} \mathrm{pc}^{-1}\right)\end{array}$ & $\begin{array}{c}\text { Direction }(\phi) \\
(\mathrm{deg})\end{array}$ & $\begin{array}{c}\mathrm{Mean}^{\mathrm{a}} \\
\left(\mathrm{km} \mathrm{s}^{-1}\right)\end{array}$ & $\begin{array}{c}\text { Number of } \\
\text { Points }\end{array}$ \\
\hline H I Hat Creek ...... & $0.289 \pm 0.003$ & $-9.6 \pm 0.7$ & $3.5 \pm 0.2$ & 37 \\
H I Effelsberg. & & & & \\
$\quad$ All points ........ & $0.338 \pm 0.001$ & $-11.1 \pm 0.2$ & $2.9 \pm 0.08$ & 268 \\
On filament ...... & $0.249 \pm 0.001$ & $-16.4 \pm 0.5$ & $2.2 \pm 0.01$ & 98 \\
Off filament ...... & $0.404 \pm 0.001$ & $1.7 \pm 0.2$ & $2.8 \pm 0.1$ & 170 \\
CO Bell Labs: & & & & \\
All points ....... & $0.4 \pm 0.2$ & $-44.0 \pm 0.2$ & $1.16 \pm 0.05$ & 453 \\
On filament ...... & $0.3 \pm 0.1$ & $-43.2 \pm 0.3$ & $0.82 \pm 0.07$ & 216 \\
\hline
\end{tabular}

NotE.-Quantities were fitted with two-dimensional $(\ell, b)$ linear least-squares. See text for details.

${ }^{a}$ Reported error is standard deviation of the mean, $\sigma / N^{1 / 2}$.

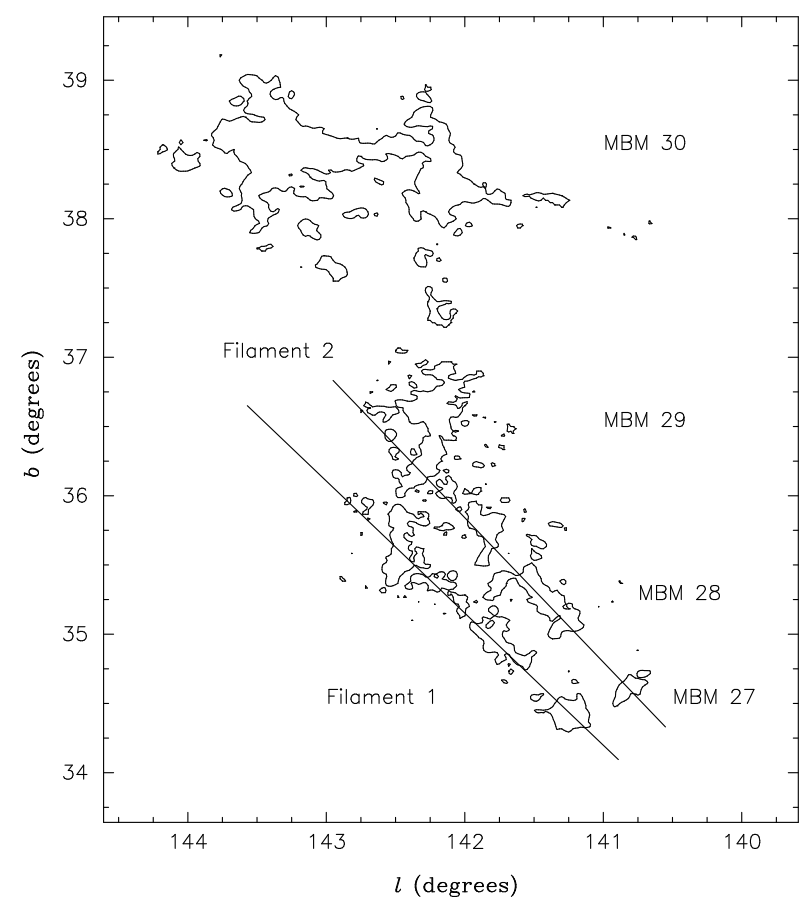

FIG. 6.-Schematic of the Ursa Major clouds. Outline is the $1.4 \mathrm{~K} \mathrm{~km}$ $\mathrm{s}^{-1}$ from Fig. 5. Solid lines indicate location of position-velocity cuts in Fig. 15. Cloud identifications from the MBM catalog are indicated.

dient is roughly perpendicular to the Galactic plane. In Figure 14, we show another representation of the $\mathrm{H}$ I centroid velocity field and the H I FWHM line width as a function of centroid velocity. The panels show these quantities "inside" and "outside" the $\mathrm{H}$ I filament. In this figure the correlated gradients in $v_{\text {cent }}(\mathrm{H} \mathrm{I})$ and $\Delta v(\mathrm{H} \mathrm{I})$ are readily apparent. Furthermore, the gradients are different "inside" and "outside" the filament, although the mean line widths are similar.

The $\mathrm{H}$ I line width gradient is not simply due to the increase in velocity components near the NCP loop because the trend begins at lower Galactic latitudes $(b \lesssim 37.5)$, where only the single profile from the sheath is present. At higher latitudes $\left(b \gtrsim 38^{\circ}\right)$, there is certainly some contribution to the overall trend from blending of the multiple components. However, in fitting Gaussian profiles to the spectra, we have endeavored to fit only the main component of the line.
To quantify the gradients apparent in the figures, we perform linear least-squares fits to $v_{\text {cent }}$ and $\Delta v$ as a function of position. All three data sets (Hat Creek, Effelsberg, and Bell Labs) have been fitted; the basic data are displayed in Figure 11, and Tables 2 and 3 summarize the results.

\subsubsection{Fitting the Gradient in $v_{\mathrm{LSR}}$}

To fit the velocity field, we used the program VFIT, which is described in detail by Goodman et al. (1993). In short, a plane is fitted to the $l-b-v$ cube, which is given by the equation

$$
v_{\mathrm{LSR}}\left(\mathrm{km} \mathrm{s}^{-1}\right)=a_{0} \ell+a_{1} b+a_{2},
$$

and each data point is weighted by the uncertainty in the determination of $v_{\mathrm{LSR}}$. To facilitate comparison, we smoothed the Bell Labs CO data to the spatial resolution of the Effelsberg $\mathrm{H}$ I data before fitting. For the Effelsberg map, we computed fits for all points in the map, for just points in the filament, and for points outside the filament (see Fig. 14). The $\mathrm{H}$ I gradients derived from the Hat Creek and Effelsberg data are in excellent agreement. The velocity gradient in the atomic gas is nearly perpendicular to the Galactic plane, and the velocity of the cloud decreases toward the plane with a magnitude of about $0.25 \mathrm{~km} \mathrm{~s}^{-1}$ $\mathrm{pc}^{-1}$. The velocity gradient determined in the "On Filament" fit is different in both magnitude and direction from the fit to all points and "Off Filament" points, which indicates that the $\mathrm{H}$ I filament is indeed a separate entity.

The fit to the $\mathrm{CO}$ data does not match well the fits to the $\mathrm{H}$ I data. While the magnitude of the $\mathrm{CO}$ velocity gradient is

TABLE 3

FitTED FWHM GRADIENTS TO H I AND CO DATA

\begin{tabular}{cccc}
\hline \hline Map & $\begin{array}{c}\text { Magnitude } \\
\left(\mathrm{km} \mathrm{s}^{-1} \mathrm{pc}^{-1}\right)\end{array}$ & $\begin{array}{c}\text { Direction } \\
(\mathrm{deg})\end{array}$ & $\begin{array}{c}\text { Weighted Mean } \\
\left(\mathrm{km} \mathrm{s}^{\mathrm{a}}\right)\end{array}$ \\
\hline H I Hat Creek: & & & \\
All points .... & $0.545 \pm 0.006$ & $5 \pm 1$ & $7.88 \pm 0.37$ \\
H I Effelsberg: & & & \\
$\quad$ All points .... & $0.423 \pm 0.001$ & $-13.0 \pm 0.4$ & $5.24 \pm 0.16$ \\
$\begin{array}{c}\text { On filament... } \\
\text { CO Bell Labs: }\end{array}$ & $0.470 \pm 0.003$ & $5.0 \pm 0.6$ & $4.14 \pm 0.18$ \\
On filament... & $0.046 \pm 0.004$ & $70 \pm 4$ & $1.32 \pm 0.03$ \\
\hline
\end{tabular}

Note.-Quantities were fitted with two-dimensional $(\ell, b)$ linear least squares. See text for details.

${ }^{a}$ Mean values are weighted by errors on individual points. Reported error is standard deviation of the mean, $\sigma / N^{1 / 2}$. 


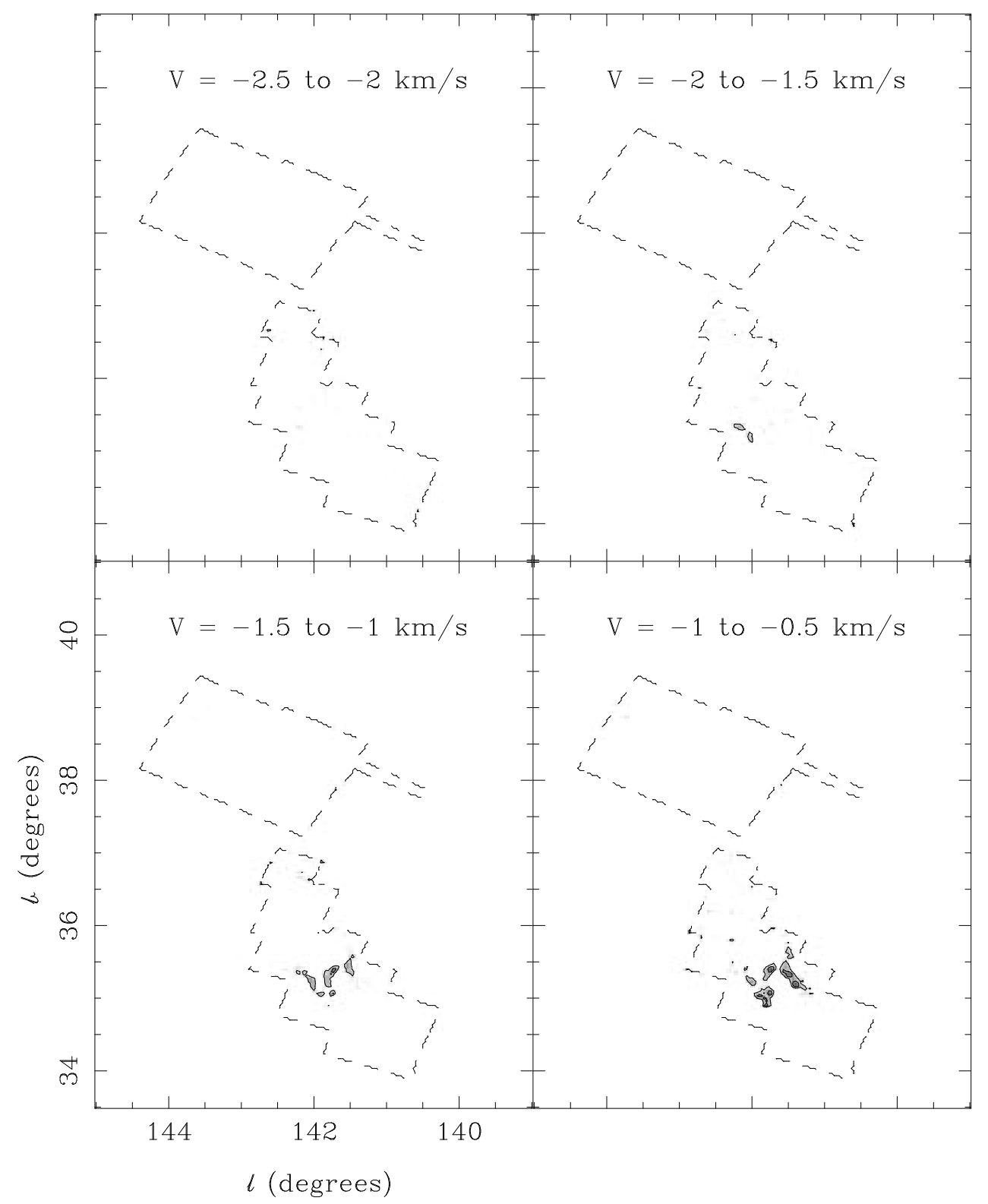

FIG. $7 a$

FIG. 7.- One-half $\mathrm{km} \mathrm{s}^{-1}$-wide channel maps of the Ursa Major complex. Contours of integrated intensity begin at $0.56 \mathrm{~K} \mathrm{~km} \mathrm{~s}^{-1}$ and increase in steps of $0.56 \mathrm{~K} \mathrm{~km} \mathrm{~s}^{-1}(3 \sigma)$. Velocity limits (LSR) of integration are indicated in each panel. The gray scale range is $0.28-2.2 \mathrm{~K} \mathrm{~km} \mathrm{~s}^{-1}$ in each panel. The data have been smoothed with a conical filter with interpolation radius $2^{\prime}$.

consistent with that of the $\mathrm{H} \mathrm{I}$, the derived direction is quite different. Both the magnitude and direction of the $\mathrm{CO}$ velocity gradient is dominated largely by filaments 1 and 2 , which affect any fit to the overall cloud. This is not surprising, since it is clear from Figures 10 and 13 that while the $\mathrm{CO}$ velocity field bears resemblance to that of the $\mathrm{H}$, it is much less organized. But most strikingly, the mean $v_{\text {cent }}(\mathrm{CO})$ is offset by $-2 \mathrm{~km} \mathrm{~s}^{-1}$ from $v_{\text {cent }}(\mathrm{H} \mathrm{I})$.

Overlaying position-velocity diagrams of the $\mathrm{H}$ I and $\mathrm{CO}$ data offers great insight into this apparent "offset" of the molecular from the atomic gas in Filaments 1 and 2. Figure 6 shows the position of two spatial cuts along the filaments, which are transformed into position-velocity diagrams in Figure 15. The centroid velocity offset between the H I (contours) and $\mathrm{CO}$ (gray scale) is quite clear and varies from 0.2 to $4.2 \mathrm{~km} \mathrm{~s}^{-1}$. The offset is about $4 \mathrm{~km} \mathrm{~s}^{-1}$ in the middle of the filaments and is close to zero at the either end. This can be seen more easily in Figure 16, which shows the atomic-molecular velocity offset versus distance along each filament. The offset is largest in MBM 28, which comprises the middle of the filaments and has the most negative $\mathrm{CO}$ velocity. Also, in filament 1 , the larger clumps seem to have a larger velocity offset, but this trend does not seem to extend to filament 2 .

\subsubsection{Fitting the Gradient in $\Delta v$}

We used a similar technique to quantify the gradients in $\Delta v$, again fitting to all the $\mathrm{H}$ I data and to "On Filament" $\mathrm{H}$ I data only. The gradient in the $\mathrm{H} \mathrm{I}$ is indeed stronger in the filament than in the surrounding medium, with a magnitude of about $0.5 \mathrm{~km} \mathrm{~s}^{-1} \mathrm{pc}^{-1}$. A weaker line width gradient may exist in the $\mathrm{CO}$, but as seen previously, the scatter 


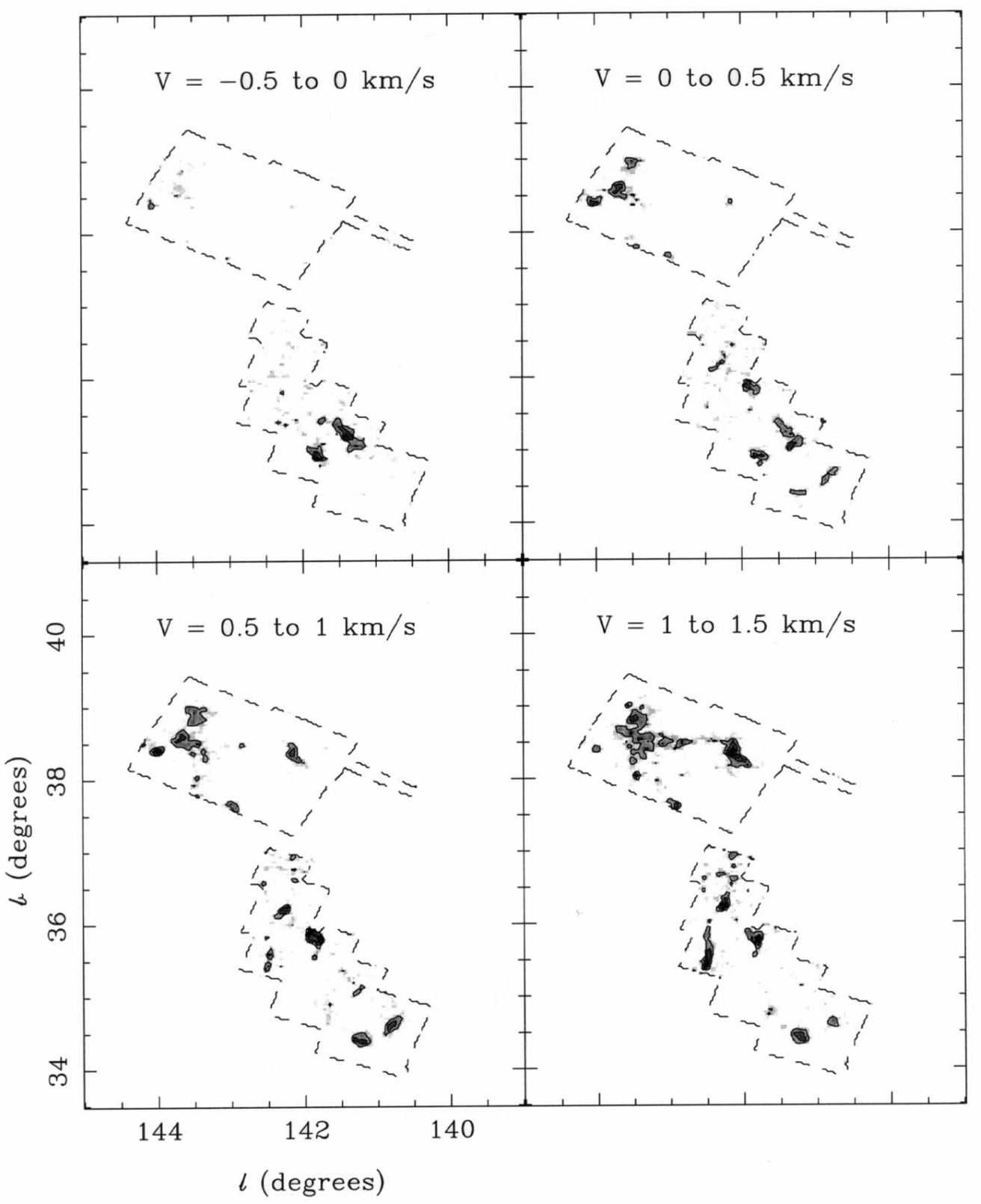

FIG. $7 b$

in the CO FWHM field is large. Higher spectral resolution observations might confirm the $\mathrm{CO}$ gradient.

\section{DISCUSSION}

\subsection{Proposed Model}

To understand the Ursa Major complex, we must develop a model that explains the morphology and velocity fields of the atomic and molecular gas, the systematic difference in the velocity centroids of the atomic and molecular gas, the line width gradient in the $\mathrm{H} \mathrm{I}$, the lack of a line width gradient in the $\mathrm{CO}$, and the arclike features seen in the IRAS and CO maps.

Meyerdierks et al. (1991) conclude that the NCP loop is a spherical or ellipsoidal bubble expanding with a velocity $v_{\exp }=10-20 \mathrm{~km} \mathrm{~s}^{-1}$. We begin by supposing that the Ursa Major clouds sit close to the surface of this bubble. At some time in the past, the atomic and molecular gases were both cospatial and comoving. Under the influence of the wind responsible for the bubble's expansion, the gas in the Ursa
Major clouds has been accelerated, but the less dense atomic gas has been accelerated more so that over time, a velocity difference has accumulated between it and the molecular gas (see Fig. 17).

If we consider a radial wind emanating from the center of the NCP loop and the Ursa Major clouds sitting somewhere near the surface of the bubble, then there are four possible locations for the clouds with respect to our line of sight. Figure 18 shows these possibilities; the clouds can be on the front or back side of the bubble and on the upper or lower half of the bubble. Depending on what portion of the bubble the clouds are located, the predicted velocity gradient and relative velocities of the atomic and molecular gases will differ.

We can immediately rule out geometries that place the clouds on the front side of the bubble (nearest us; quadrants 1 and 4 in Fig. 18) because expansion of the NCP bubble would make the dense molecular gas appear redshifted with respect to the lower density atomic gas - the opposite of what we observe. In quadrants 2 and 3, on the back side of 


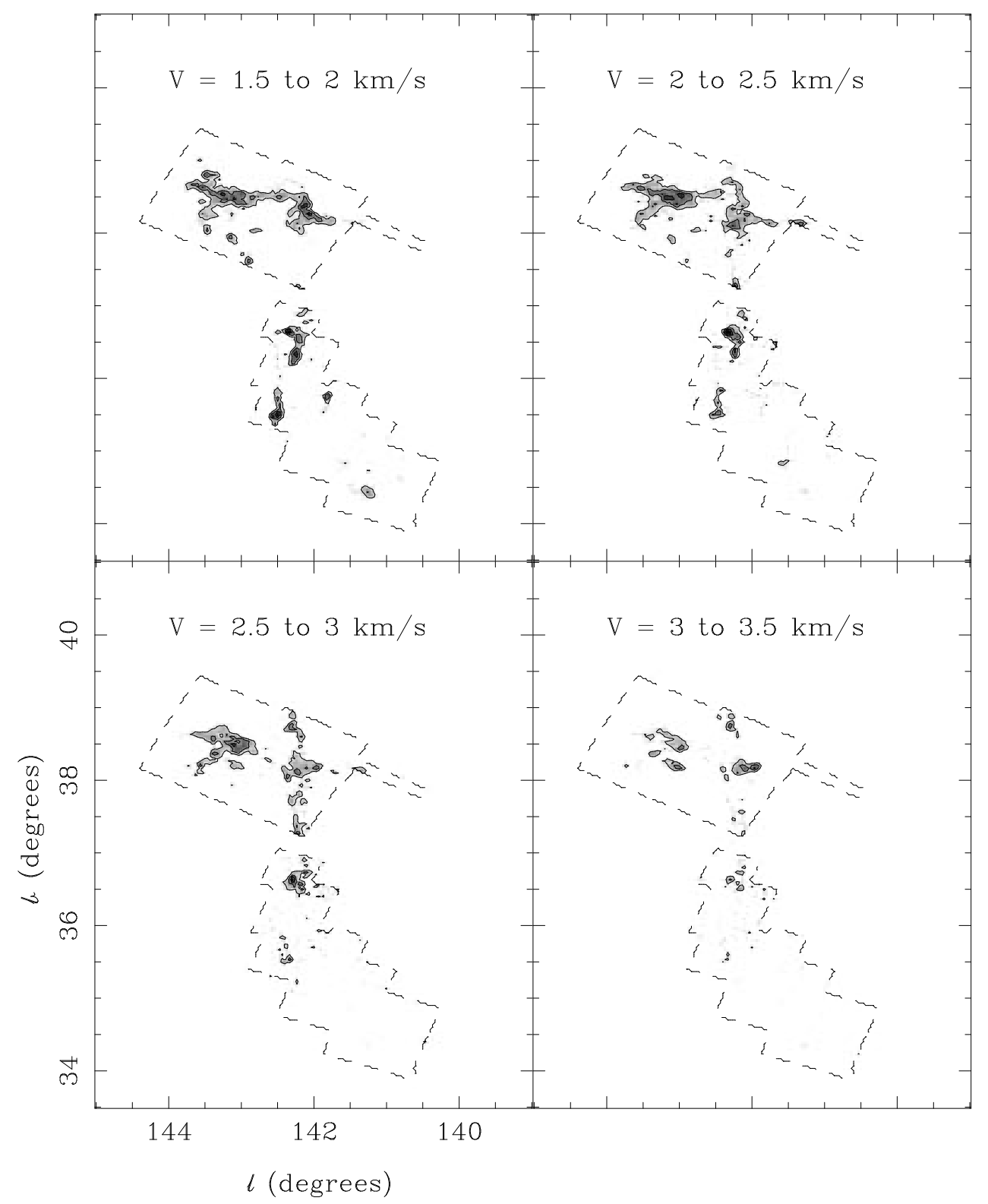

FIG. $7 c$

the bubble, the velocity shift has the same sense as what is observed; the molecular gas is blueshifted with respect to the atomic gas. Furthermore, if the clouds are on the back side of an expanding bubble, their LSR velocity should be more positive than the rest velocity of the bubble. This is indeed the case-Meyerdierks et al. (1991) report the rest velocity of the bubble to be between -2 and $-4 \mathrm{~km} \mathrm{~s}^{-1}$ LSR, some 6-8 $\mathrm{km} \mathrm{s}^{-1}$ blueward of the $\mathrm{H}$ I filament centroid velocity. So, the Ursa Major clouds must be on the back side of the NCP bubble.

In quadrant 3 , expansion would produce an LSR velocity gradient such that the gas appears more blueshifted toward lower Galactic latitude, which is consistent with our observations. However, the clouds do not look as if they are in the lower quadrant of the bubble - they appear to be at the top of the NCP loop (quadrant 2). (Indeed, if the clouds are in quadrant 3 , then the three-dimensional shape of the bubble is rather peculiar.) Furthermore, the IRAS and $\mathrm{CO}$ arcs have upward-pointing "tails" (toward higher $b$ ), which is difficult to understand if the wind were blowing downward onto the clouds, as it would have to be if the clouds were in quadrant 3. For these reasons, it is unlikely that the clouds are in quadrant 3.

In quadrant 2 , however, the apparent $v_{\mathrm{LSR}}$ gradient due to expansion would have the opposite sign from quadrant 3 and therefore the opposite sign from what we observe. But if the cloud complex as a whole were also undergoing moderate rotation in a direction away from us and toward the Galactic plane, it would be enough to change the sense of the predicted $v_{\text {LSR }}$ gradient and make it consistent with our observations. Thus, considering both the expansion and rotation, the observed velocity gradient, $\nabla v_{\text {tot }}$, is given by

$$
\nabla v_{\text {tot }}=\nabla v_{\text {exp }}+\nabla v_{\text {rot }} \text {. }
$$

So, given the sign of the observed velocity gradient, the viable alternatives are as follows: 


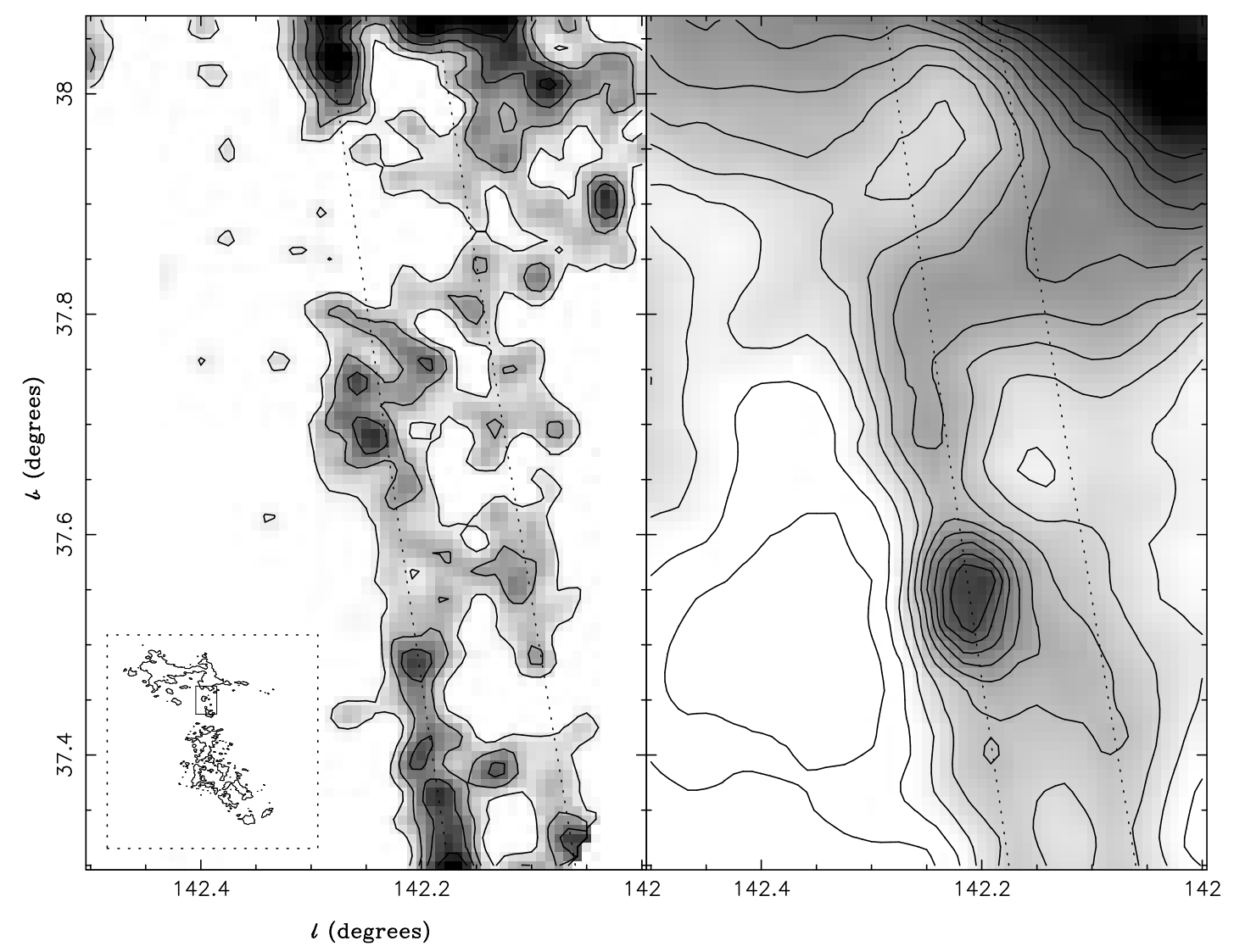

FIG. $8 a$

FIG. 8.-Examples of the fine-scale filaments seen in CO. The insets are finding charts (essentially miniature versions of Fig. 6) with boxes indicating the location of the filaments in the CO cloud. (a) Indicated by dotted lines are two CO filaments which are parallel to within $1^{\circ}(l e f t$-hand panel). Their widths are barely resolved by our observations, and their aspect ratios are at least 15 . Contours of integrated intensity between $v_{\mathrm{LSR}}=1-3.75 \mathrm{~km} \mathrm{~s}{ }^{-1}$ are shown at 0.78 , $1.56, \ldots, 6.2 \mathrm{~K} \mathrm{~km} \mathrm{~s}^{-1}$; gray scale is $0.52-3.2 \mathrm{~K} \mathrm{~km} \mathrm{~s}^{-1}$. The $\mathrm{CO}$ data have been smoothed with a conical filter with interpolation radius 1.5. Right-hand panel is the IRAS $100 \mu \mathrm{m}$ image of the same region. The resolution of the IRAS image is coarser than the CO image, but a hint of the double filaments can still be seen. An unresolved $100 \mu \mathrm{m}$ source at $(142.2,34.55)$ has no counterpart in our CO maps. Contours are shown at $2.0,2.4, \ldots, 6 \mathrm{MJy}^{-1}$; gray scale is 2.5-7 $\mathrm{MJy} \mathrm{Sr}^{-1}$. (b) Another straight $\mathrm{CO}$ filament in which the clumps appear as "beads on a string" (right-hand panel). Again the aspect ratio of this filament is at least 15 . Contours of integrated intensity between $v_{\mathrm{LSR}}=0-3 \mathrm{~km} \mathrm{~s}^{-1}$ are shown at $0.67,1.33, \ldots, 5.33 \mathrm{~K} \mathrm{~km} \mathrm{~s} \mathrm{~km}^{-1}$; gray scale is $0.39-7.8 \mathrm{~K} \mathrm{~km} \mathrm{~s}{ }^{-1}$. The $\mathrm{CO}$ data have been smoothed with a conical filter with interpolation radius $2^{\prime}$. Right-hand panel is the IRAS $60 \mu \mathrm{m}$ image of the same region. Contours are shown at $0.375,0.5, \ldots, 1.25 \mathrm{MJy} \mathrm{Sr}^{-1}$; gray scale is $0.5-1.25 \mathrm{MJy} \mathrm{Sr}^{-1}$.

1. The clouds are in quadrant 3 and are being "pushed" away from us by the expansion of the NCP loop. In this case, it is possible to assume that $\left|\nabla v_{\text {rot }}\right|=0$.

2. The clouds are in quadrant 2 and have a global rotational motion (for instance, "sliding" down the back side of the bubble) in addition to being "pushed" by the expansion of the NCP loop. In this case, we must assume that $\left|\nabla v_{\text {rot }}\right| \neq 0$.

Again, because of the morphological evidence, we believe that scenario (1) is improbable and that scenario (2) is the correct interpretation.

\subsection{Model Predictions and Tests}

\subsubsection{Velocity Gradients: Expansion and Rotation}

Assume a spherical bubble of radius $R$ expanding with velocity $v_{\text {exp }}$. An observer would measure the line-of-sight component of this motion $v_{\exp , z}=v_{\exp } \cos \theta$, where $\theta$ is the angle between the $z$-axis and the point on the sphere being observed and $\theta=0$ corresponds to a line of sight directly through the center of the bubble $(z=0)$. The $y$-component of the gradient in $z$-velocity due to expansion is

$$
\begin{aligned}
\nabla_{y} v_{\exp } & =\frac{\partial}{\partial y}\left(v_{\exp } \cos \theta\right) \\
& =\frac{v_{\exp }}{R} \frac{\partial}{\partial y}\left(R-y^{2}\right)^{1 / 2} \\
& =-\frac{v_{\exp } y}{R}\left(R^{2}-y^{2}\right)^{-1 / 2} .
\end{aligned}
$$

The $y$-component of the gradient due to solid body rotation at an angular velocity $\omega$ is

$$
\nabla_{y} v_{\text {rot }}=\omega \cos \phi
$$

where $\phi$ is the angle between the velocity gradient on the plane of the sky and the $y$-axis. Thus, the total observed $v_{\text {LSR }}$ gradient along the $y$-axis is

$$
\nabla_{y} v_{\text {tot }}=-\frac{v_{\text {exp }} y}{R}\left(R^{2}-y^{2}\right)^{-1 / 2}+\omega \cos \phi .
$$




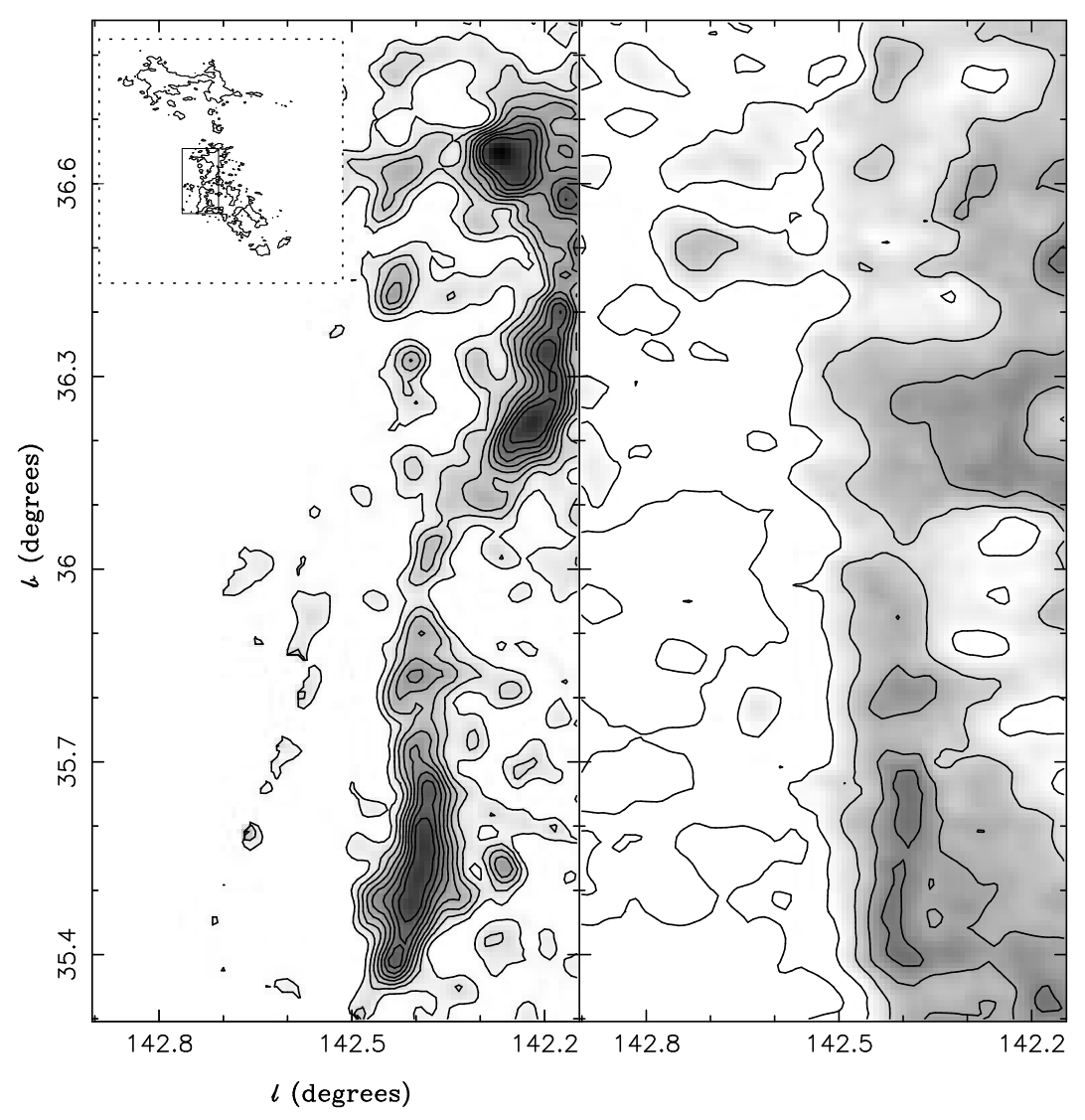

FIG. $8 b$

Obviously, since solid body rotation produces a constant velocity gradient (see eq. [8]), linear fits to velocity versus position along the gradient axis suffice when one is only concerned with rotation (see, e.g., Goodman et al. 1993). In the case of expansion, however, equation (7) shows that the gradient in velocity with position is not constant. However, we can estimate how much of a gradient a linear fit to equation (7) would find, if we know the total angular extent $\Delta \theta$ from one end of the filament to the other.

Using $v_{\exp }=15 \mathrm{~km} \mathrm{~s}^{-1}$ and $R=30 \mathrm{pc}$-numbers appropriate to the NCP loop-we have performed linear fits to equation (7) to determine $\nabla_{y} v_{\exp }$ over a variety of ranges for $\theta$. The gradient due to rotation $\nabla_{y} v_{\text {rot }}$ is then the "additional" gradient needed to reproduce the observed gradient, $\left|\nabla_{y} v_{\text {tot }}\right| \sim 0.3 \mathrm{~km} \mathrm{~s}^{-1} \mathrm{pc}^{-1}$. The results are shown in Table 4. For instance, assuming the clouds are in quadrant 2 , then $\nabla_{y} v_{\exp } \sim-0.3 \mathrm{~km} \mathrm{~s}^{-1} \mathrm{pc}^{-1}, 30 \leq \theta \leq 90$, and $\nabla_{y} v_{\text {rot }} \sim 0.6 \mathrm{~km} \mathrm{~s}^{-1} \mathrm{pc}^{-1}$ (toward the Galactic plane) can reproduce the observed $\left|\nabla_{y} v_{\text {tot }}\right|$. The linear extent of the clouds along the surface of the bubble would then be $L=2 \pi R\left(\Delta \theta / 360^{\circ}\right) \sim 31 \mathrm{pc}$. Note this would make the dis- tance to MBM 28 about $140 \mathrm{pc}$, which is still consistent with Penprase's (1993) upper limit.

Assuming a Galactic midplane density of $\rho_{0}=10^{-23} \mathrm{~g}^{-1}$ $\mathrm{cm}^{-3}$, we would expect a velocity gradient along the $\sim 10$ pc length of the filaments of about $0.2 \mathrm{~km} \mathrm{~s}^{-1} \mathrm{pc}^{-1}$, due simply to free fall toward the Galactic disk. This gradient is of the same order of magnitude as the $\nabla_{y} v_{\text {rot }}$ required to reproduce the observed velocity gradient (Table 4). However, motion directly toward the Galactic plane would not have any component along our line of sight. To give rise to a line-of-sight velocity component, some mechanism is necessary to constrain the clouds to "slide down" along the back (curved) surface of the bubble. Such a mechanism might be the Parker instability wherein hot gas from the interior of the bubble provides upward pressure on the horizontal component of the Galactic magnetic field, which results in a loop of field lines out of the Galactic disk. Gas threaded by the magnetic field (in our case, the Ursa Major clouds) will eventually slide down along the field lines under the gravitational attraction of the Galactic disk, following the surface contour of the bubble (see, e.g., Kamaya et al.

TABLE 4

Some Predicted Velocity Gradients

\begin{tabular}{cccccc}
\hline \hline Model & $\begin{array}{c}\text { Range of } \theta \\
(\mathrm{deg})\end{array}$ & $\begin{array}{c}\Delta \theta \\
(\mathrm{deg})\end{array}$ & $\begin{array}{c}\nabla_{y} v_{\text {exp }} \\
\left(\mathrm{km} \mathrm{s}^{-1} \mathrm{pc}^{-1}\right)\end{array}$ & $\begin{array}{c}\nabla_{\underline{y}} v_{\text {rot }} \\
\left(\mathrm{km} \mathrm{s}^{-1} \mathrm{pc}^{-1}\right)\end{array}$ & $\begin{array}{c}L^{\mathrm{a}} \\
(\mathrm{pc})\end{array}$ \\
\hline Pure expansion ............. & $-30 \leq \theta \leq-90$ & 60 & 0.3 & 0.0 & 31 \\
Expansion + rotation...... & $30 \leq \theta \leq 90$ & 60 & -0.3 & 0.6 & 31 \\
& $45 \leq \theta \leq 90$ & 45 & -0.2 & 0.5 & 24 \\
& $68 \leq \theta \leq 90$ & 22 & -0.1 & 0.4 & 12 \\
\hline
\end{tabular}

NoTE. - The total measured velocity gradient, $\nabla_{y} v_{\text {tot }}=\nabla_{y} v_{\text {exp }}+\nabla_{y} v_{\text {rot }}$, is about $0.3 \mathrm{~km} \mathrm{~s}^{-1} \mathrm{pc}^{-1}$.

${ }^{\text {a }}$ Linear extent of clouds for given $\Delta \theta$. 


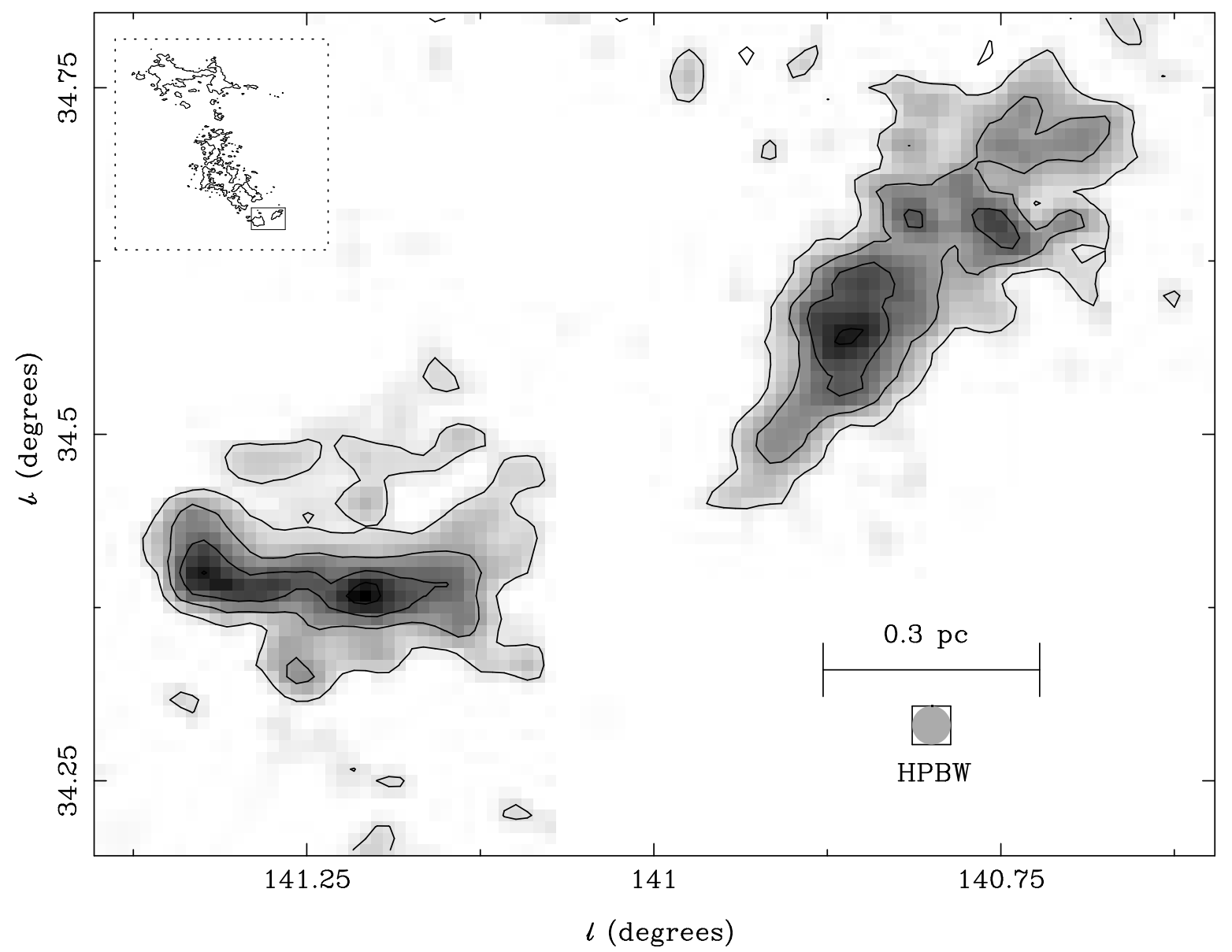

FIG. 9.-An "upward-pointing" $\mathrm{CO}$ arc at the tips of Filaments 1 and 2. The inset is a finding chart with box indicating the location of this feature in the $\mathrm{CO}$ cloud. Contours are integrated intensity between $v_{\mathrm{LSR}}=0-0.75 \mathrm{~km} \mathrm{~s}^{-1}$ shown at $-0.5,0.5, \ldots, 2.5 \mathrm{~K} \mathrm{~km} \mathrm{~s}^{-1}$. The gray scale ranges from 0.28 to $2.2 \mathrm{~K}$ $\mathrm{km} \mathrm{s}^{-1}$. The data have been smoothed with a conical filter with interpolation radius 1.2. The orientation of the arc can be used to discriminate between possible locations of the cloud complex with respect to the NCP bubble. Since the arc points toward higher $b$, we conclude the clouds are on the upper hemisphere of the bubble (see text and Fig. 18).

1996). We believe the Parker instability scenario to be a plausible explanation for $\nabla_{y} v_{\text {rot }}$, but little is known about the three-dimensional magnetic field structure in the NCP loop (see $\S 4.2 .3)$.

\subsubsection{Systematic Velocity Offsets}

In $\S 3.3$, we noted that the $\mathrm{H} \mathrm{I}$ and $\mathrm{CO}$ centroid velocities differ by $2-4 \mathrm{~km} \mathrm{~s}^{-1}$, with the molecular gas blueshifted with respect to the atomic (see Figs. 15 and 16). Such an offset in atomic and molecular centroid velocities can arise from $\mathrm{H}$ I self-absorption. We view this possibility as unlikely in Ursa Major because the $\mathrm{H}$ I line profiles in the filament are quite symmetric and, as mentioned previously, the line cores are Gaussian.

We might expect that the difference in centroid velocity between the $\mathrm{H}$ I and $\mathrm{CO}$ should be correlated with clump density, with the denser clumps showing a larger offset in $v_{\text {cent }}$. The low-mass, less dense clumps would be carried along with the atomic gas by the wind, while denser clumps would be "left behind." If the quantity $I\left({ }^{12} \mathrm{CO}\right) A$ is proportional to mass, then the volume density is roughly proportional to $I\left({ }^{12} \mathrm{CO}\right) A^{1 / 2}$, where $A$ is the projected area of the clump. We took values of the atomic-molecular velocity offset $\left[v_{\text {cent }}(\mathrm{H} \mathrm{I})-v_{\text {cent }}(\mathrm{CO})\right]$ from the position-velocity diagrams in Figure 15 and values of $I\left({ }^{12} \mathrm{CO}\right)$ and $A$ from Figure 5. Plotting velocity offset versus scaled density $I\left({ }^{12} \mathrm{CO}\right) A^{1 / 2}$, we found no obvious trend. However, the scaled density varies only by a factor of 2.5 , which may be too small to see a trend. Also, such a comparison implicitly assumes that the conversion factor between $I\left({ }^{12} \mathrm{CO}\right) A$ and mass (the so-called $X$ factor; see, e.g., Bloemen et al. 1986) is constant. If the $X$ factor varied from clump to clump, the variation could erase from our data any trend of velocity offset with $I\left({ }^{12} \mathrm{CO}\right) A^{1 / 2}$. A better determination of clump density through multispecies or multitransition observations might aid further investigation of this expected correlation.

We might also expect that clumps with either large surface area or low density (or both) would be accelerated more by the wind than high-density, low surface area clumps. To test this idea, we examined the relationship between the atomic-molecular velocity offset and clump area divided by scaled density, assumed to be proportional to $A^{3 / 2} / I\left({ }^{12} \mathrm{CO}\right.$ ) (but subject to the same $X$-factor caveat). Again, we found no trend, but again there is little dynamic range in $A^{3 / 2} / I\left({ }^{12} \mathrm{CO}\right)$. Finally, the clump cross sections "seen" by the wind are likely to be different from the ones we see, particularly since the cloud is so filamentary, so our values for $A$ may not be appropriate.

\subsubsection{Line Width Gradients}

We now turn to the line width gradient seen in the $\mathrm{H} \mathrm{I}$. We consider three possible explanations for this below, but we favor the explanation in which the gradient is produced 

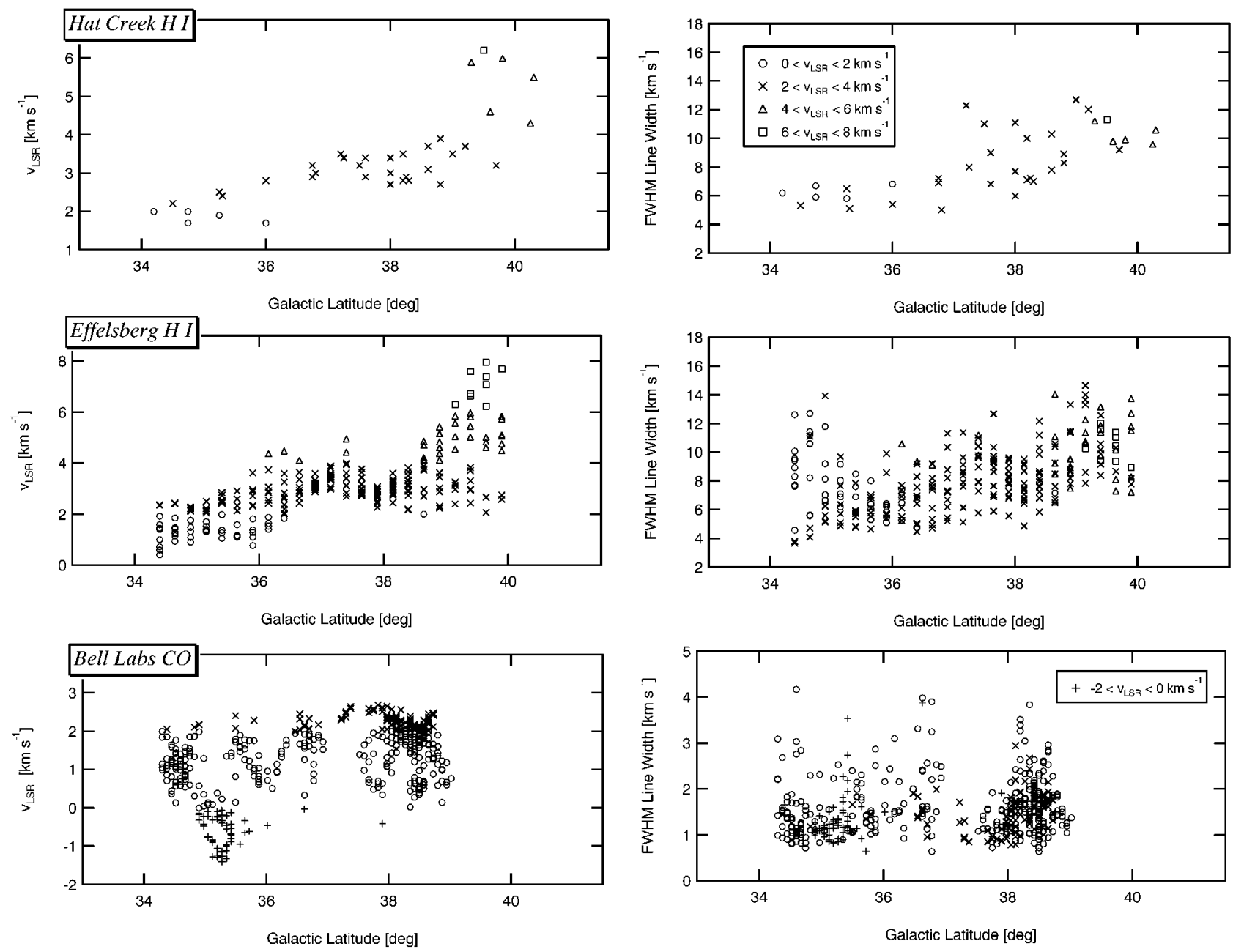

Fig. 11.-Kinematic properties of the atomic and molecular gas as a function of Galactic latitude. The different symbols represent the specified velocity ranges in all panels. Top panels: H I centroid velocity and FWHM line width from Hat Creek. Middle panels: $\mathrm{H}$ i centroid velocity and FWHM line width from Effelsberg. Bottom panels: CO centroid velocity and FWHM line width from Bell Labs. The CO spectra were spatially smoothed to the Effelsberg resolution before their velocities and line widths were measured.

by directional anisotropy in the velocity dispersion (explanation [2], below).

1. Increase in the three-dimensional velocity dispersion.-It may be that the turbulence in the gas increases with $b$, increasing the nonthermal, and thus the total, line width. Ferriere, Mac Low, \& Zweibel (1991) have modeled an expanding superbubble in a uniform magnetic field and report that magnetic pressure is expected to increase off the axis of the field. The increase in magnetic pressure is proportional to $\sin ^{2} \theta$ (see eqs. [41] and [42] of Ferriere et al.). Thus, if the clouds extend over $30^{\circ} \geq \theta \geq 90^{\circ}$, the magnetic pressure would increase by a factor of 4 from the bottom to the top of the clouds, assuming the line-of-sight thickness through the clouds is roughly constant. If the volume density of the gas stays constant, then a fourfold increase in pressure would cause a twofold increase in the gas velocity dispersion and hence in the line width - very close to what we observe in the $\mathrm{H}$ I data. There are a few caveats to this explanation. For the Ferriere et al. model to apply to the Ursa Major complex, the external magnetic field must have a component parallel to the Galactic plane and along our line of sight. Furthermore, we would expect the pressure in the molecular clumps to increase in order to maintain pressure equilibrium. However, we see no evidence of a line width increase in the CO. Alternatively, the density in the clumps would need to increase by a factor of about 4 if the $\mathrm{CO}$ line width were to remain unchanged. Observations of the clumps in multiple transitions of molecular species with higher dipole moments (e.g., $\mathrm{CS}, \mathrm{HCN}$ ) that trace higher densities might confirm an increase in density. (However, an increase as small as a factor of 4 may be difficult to measure.)

2. Anisotropic velocity dispersion.-It is possible that the three-dimensional velocity dispersion is anisotropic and thus that the observed line width gradient is produced by the changing inclination angle of a curved filament with respect to the line of sight. This is illustrated in Figure 19. Assume that the measured line width in the $\mathrm{H}$ I filament (that is, the component of the velocity dispersion along our line of sight) can be decomposed into a "tangential" component $\Delta v_{\tan }$ that runs along the filament's length and a "radial" component $\Delta v_{\text {rad }}$ that runs across it, radial to the expanding bubble. In quadrant 2 , lines of sight toward the lower $b$ will measure primarily $\Delta v_{\text {rad }}$, while those toward the higher $b$ will measure primarily $\Delta v_{\tan }$. In quadrant 3 , the situation is reversed; $\Delta v_{\mathrm{tan}}$ dominates at lower $b$ and $\Delta v_{\mathrm{rad}}$ at higher $b$. Our $\mathrm{H}$ I measurements show that $\Delta v$ increases with $b$, so, for an anisotropic line width explanation to 


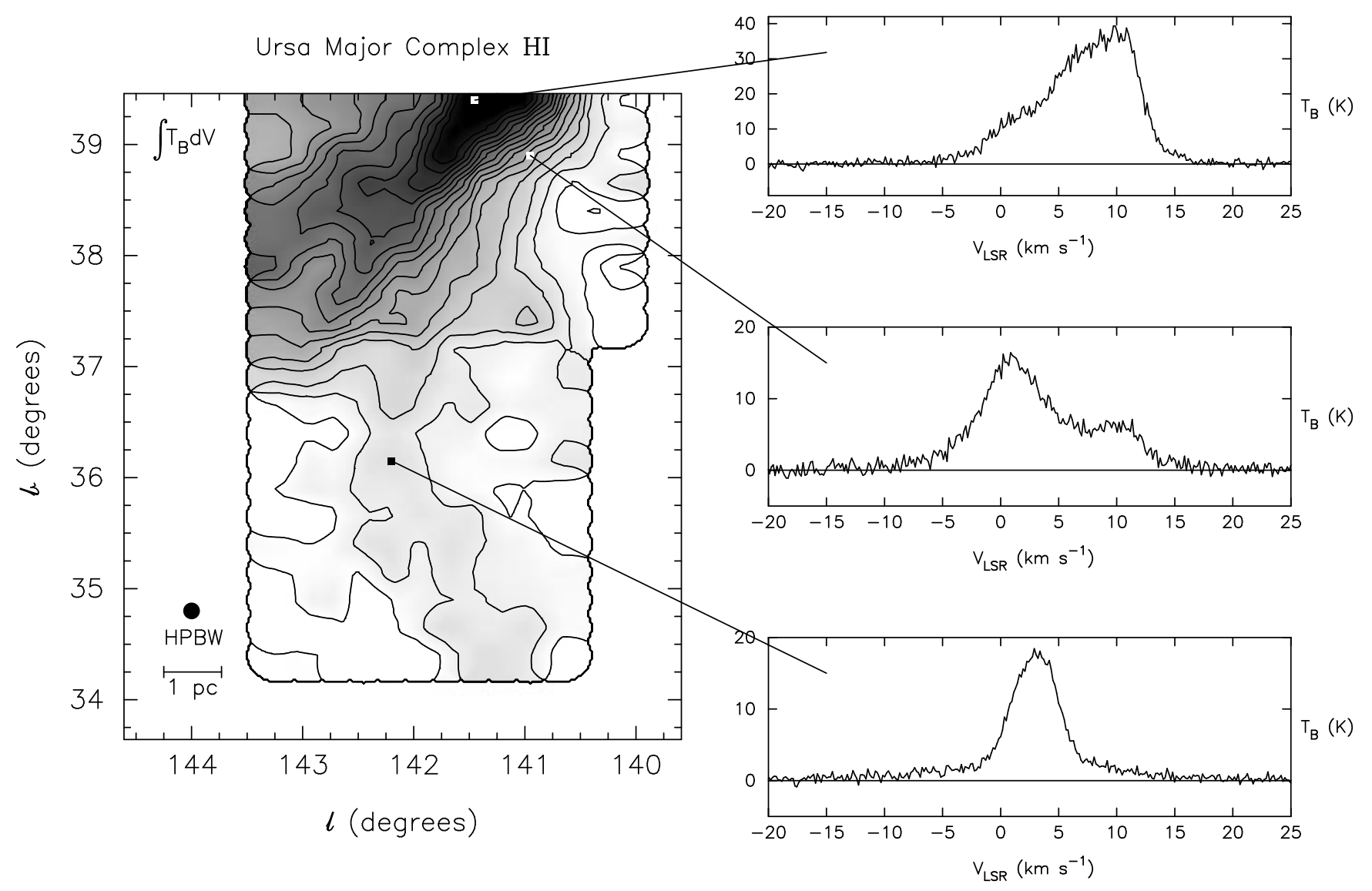

FIG. 12.-Integrated intensity of $\mathrm{H}$ I from $v_{\mathrm{LSR}}=-20$ to $20 \mathrm{~km} \mathrm{~s}^{-1}$. Contours are shown at $15,30, \ldots, 300 \mathrm{~K} \mathrm{~km} \mathrm{~s}^{-1}$, and the gray scale ranges from 90 to $320 \mathrm{~K} \mathrm{~km} \mathrm{~s}^{-1}$. Representative spectra are shown from points indicated. In the $\mathrm{H}$ I filament, the spectra are simple with Gaussian cores. Outside the filament, the profiles are more complex.

apply, $\Delta v_{\text {tan }} \gg \Delta v_{\text {rad }}$ if the clouds are in quadrant 2 , or $\Delta v_{\text {rad }} \gg \Delta v_{\text {tan }}$ if they are in quadrant 3 (Fig. 19).

This kind of line width anisotropy can be produced in a region pervaded by dynamically significant magnetic fields. In such regions, the forces felt by particles moving along and across field lines are not the same. For example, if the field direction is parallel to the long axis of a filament, then the line width perpendicular to the filament should be similar to the Alfvén speed, $v_{\mathrm{A}}$, but the line width along the filament need not be $\sim v_{\mathrm{A}}$. The line width along the filament is limited by mass motion along field lines and/or by magnetosonic waves. Recall that in the scenario in which the Ursa Major filament "hangs down" from the top of the NCP Loop (quadrant 2), the data require that $\Delta v_{\text {rad }} \gg \Delta v_{\text {tan }}$ in order for an anisotropic line width to explain the observed line width gradient. In the case of a field along the filament, this means that some process creates a line width along the filaments that is larger than the Alfvén speed. We can imagine that such a situation could arise if streaming motion of gas along field lines creates a large line width along the filament. Unfortunately, the only existing indication of the plane-of-the-sky field structure in Ursa Major comes from a small optical polarization map (Seki 1990) that does not cover the filamentary clouds, so we can say little about the true direction of the field with respect to the filaments.

Note that the anisotropic line width explanation does not require the presence of a dynamically significant magnetic field. For example, it is easy to believe that motions along the surface of a bubble interior to the NCP Loop are not of the same magnitude as the motions perpendicular to the bubble surface.

That said, though, we should point out that two independent lines of evidence point to a dynamically significant magnetic field pervading the Ursa Major clouds. First, extensive observations of the $\mathrm{H}$ I Zeeman effect have shown that the observed line widths are similar to the Alfvén speed throughout the region (Myers et al. 1995). And second, a comparison of column density and field strength shows that these two quantities are correlated in the filamentary portion of the clouds but not outside the filaments. This comparison is presented in Figure 20, which shows that in the filaments the line-of-sight magnetic field, $B_{z}$, determined from Zeeman observations is extremely well correlated with $I(100)$, which is roughly proportional to column density (lower panel). The self-gravitational energy in the Ursa Major clouds is insignificant compared to their magnetic energy (Myers et al. 1995), so the fact that field strength and column density are proportional to each other in the filaments can surely not be explained by the classic arguments inwhich clouds are magnetically supported against their own gravity (see, e.g., Elmegreen 1987). Instead, we suggest that the correlation of field strength and column density in the filament (and not off the filament) is the result of a dynamical process which compresses both gas (producing the filaments) and field lines. Notice that this interpretation 


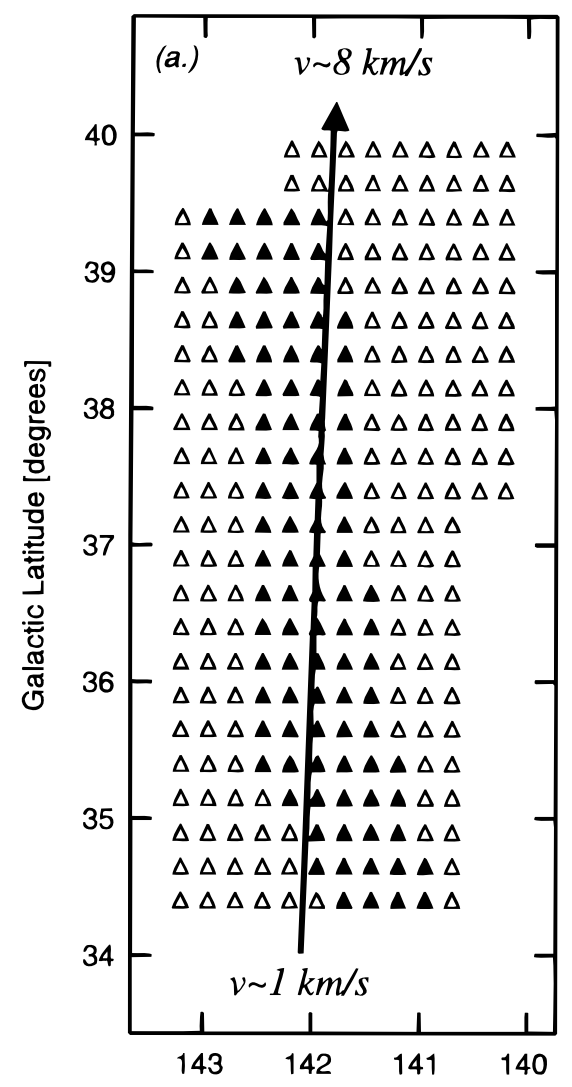

Galactic Longitude [degrees]

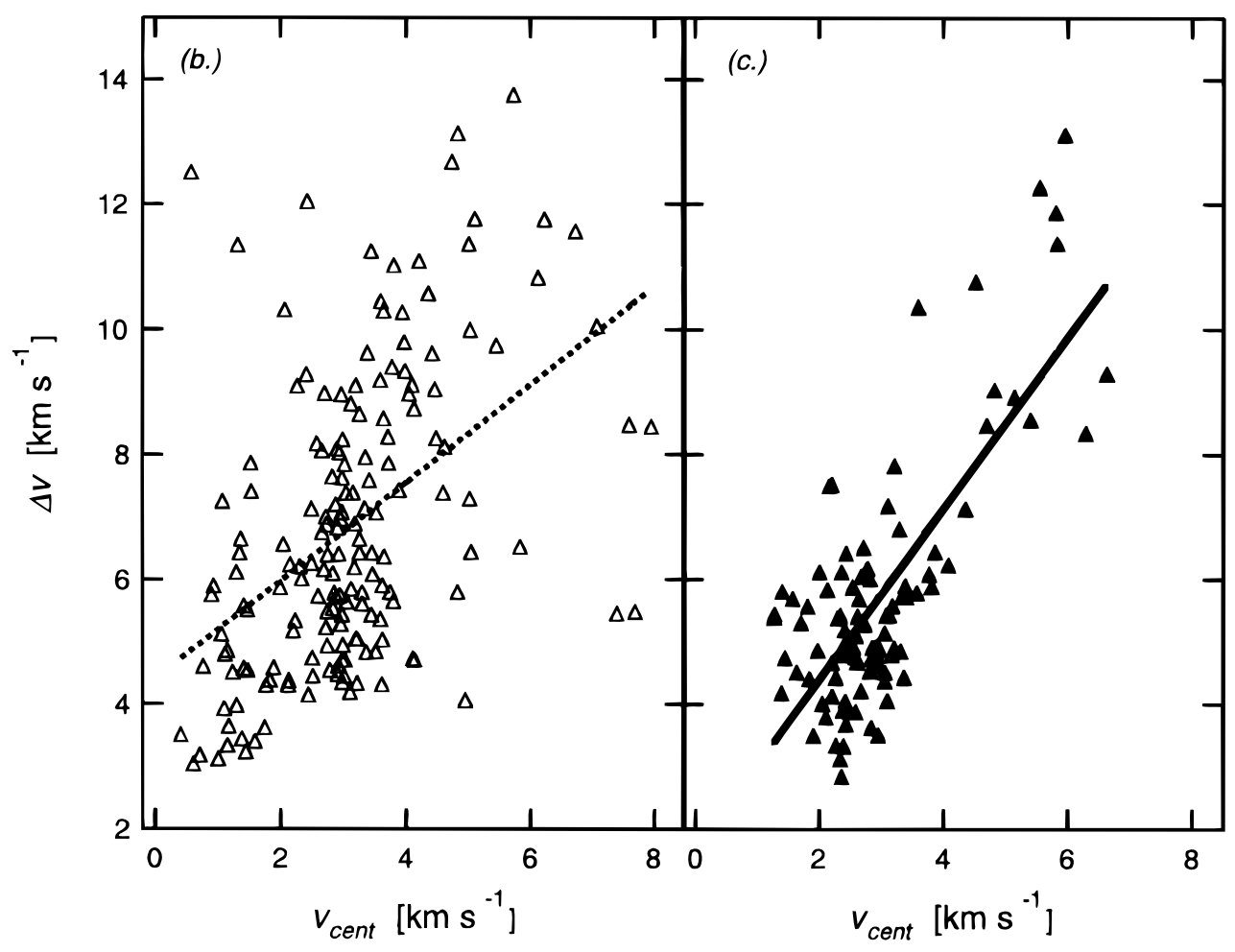

FIG. 14.-The $\mathrm{H}$ I velocity field inside and outside of the atomic filament. (a) Filled triangles indicate position of spectra associated with the $\mathrm{H}$ i filament, and open triangles are "nonfilament" spectra. The arrow indicates the direction of the fitted velocity gradient in the H I filament. Panels $(b)$ and $(c)$ show $\mathrm{H}$ I centroid velocity vs. $\mathrm{H}$ I line width for the two regions with linear fits indicated. See Tables 2 and 3. 

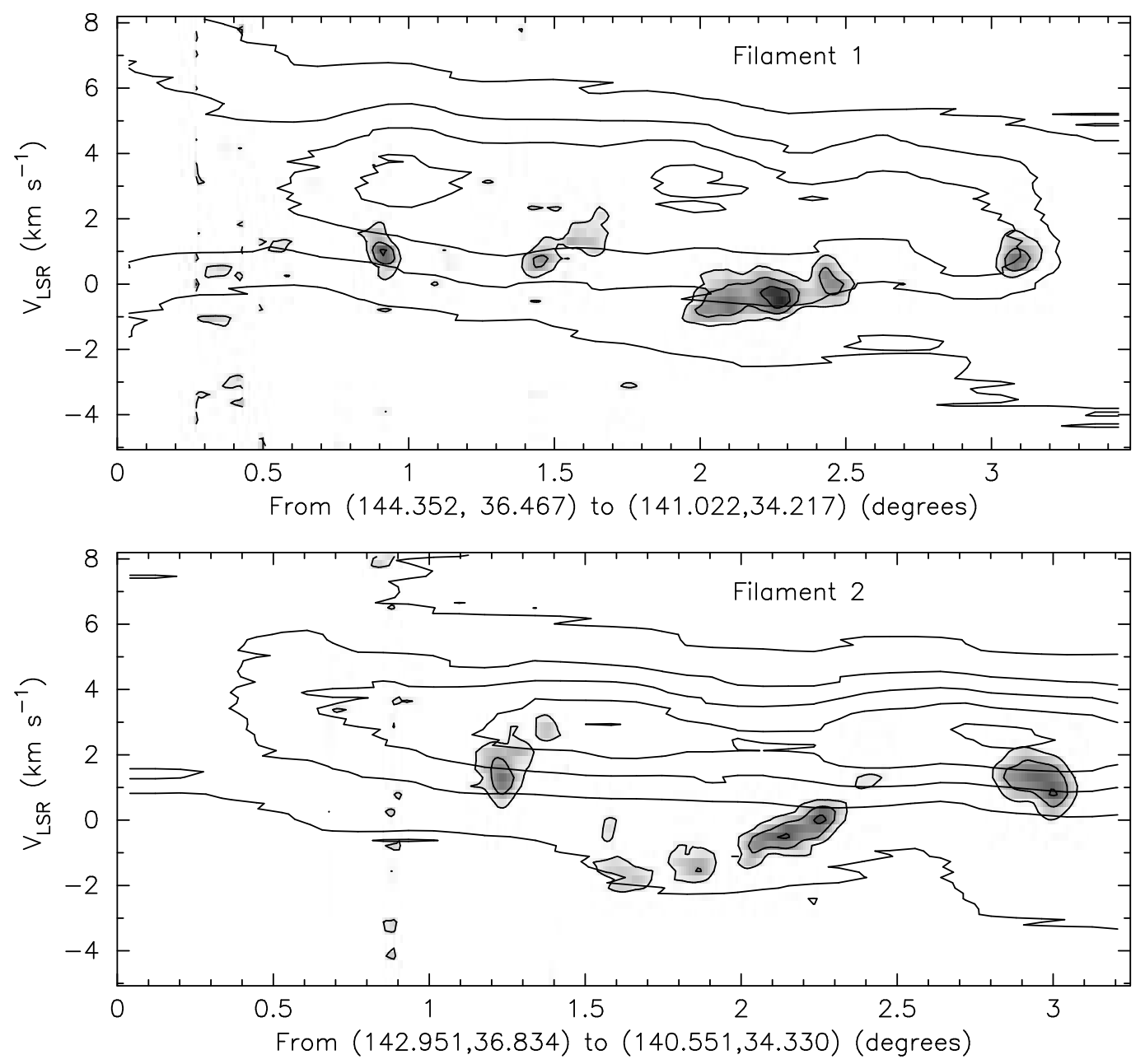

FIG. 15.-Position-velocity cuts of $\mathrm{H} \mathrm{I}$ (contours) and CO (gray scale) in Filament 1 (upper panel) and Filament 2 (lower panel). See Fig. 6 for the orientation of the cuts. The $\mathrm{CO}$ and $\mathrm{H}$ I centroid velocities differ by up to $4 \mathrm{~km} \mathrm{~s}^{-1}$, with the CO blueshifted with respect to the $\mathrm{H}$ I. This is evidence that the clouds have interacted with the wind, causing the expansion of the NCP loop. $\mathrm{H}$ I contours are at 4, 8, ., $20 \mathrm{~K}$ and $\mathrm{CO}$ contours are at $1,2,3 \mathrm{~K}$. The CO data have been smoothed with a conical filter with interpolation radius $3^{\prime}$.

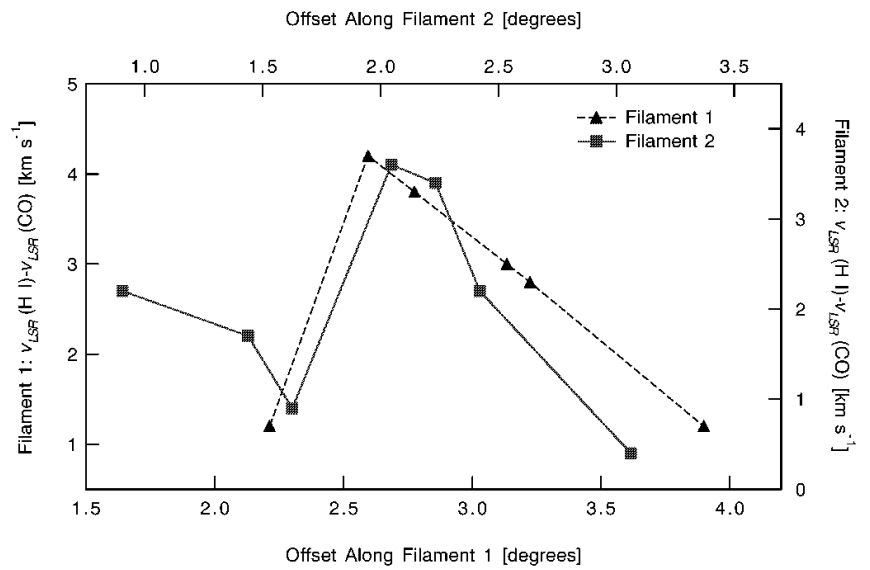

FIG. 16. - Centroid velocity offsets in the filaments as a function of position. Filament 1 utilizes the left and bottom axes, and Filament 2 utilizes the right and top axes. The velocity offsets axes show the LSR velocity of the $\mathrm{H}$ I minus the LSR velocity of the CO at various "clump" positions along the filaments (see Figs. 6 and 15). Note how the deviations of the $\mathrm{CO}$ from the $\mathrm{H}$ I velocity are maximal toward the "middle" of the filaments and minimal toward the "ends." The zero points of the comparable (e.g., top and bottom) axes are offset to align the curves, but the absolute offset and velocity scales are the same for both filaments. requires only that the line-of-sight component of the field be compressed, as that is all that is measurable in the Zeeman observations.

3. Increase in the number of "clumps" toward high latitude.-It may be that there are simply more clumps along the line of sight through the top of the filament than through the bottom. However, along the entire length of the filament the $\mathrm{H}$ I line shapes remain simple Gaussian cores with broad wings. If there were more clumps along the line of sight through the top of the filament, we might expect some departure from this simple shape. Furthermore, the $\mathrm{CO}$ position-velocity diagrams along filaments 1 and 2 (Fig. 15) do not show more molecular clumps at higher $b$.

\subsubsection{IRAS Colors}

In $\S 3.2$, we found that in the tips of filaments 1 and 2 , the mean IRAS temperature $T(12 / 25)$ increases by about $15 \%$ and mean color ratio $R(12 / 25)$ increases by about $50 \%$ above the mean quantities in the other parts of the filaments. Previous authors have concluded that changes in $I R A S$ colors such as $R(12 / 25)$ reflect changes in the abundance of small particles. Boulanger et al. (1990) studied four molecular clouds including Ursa Major and found that 


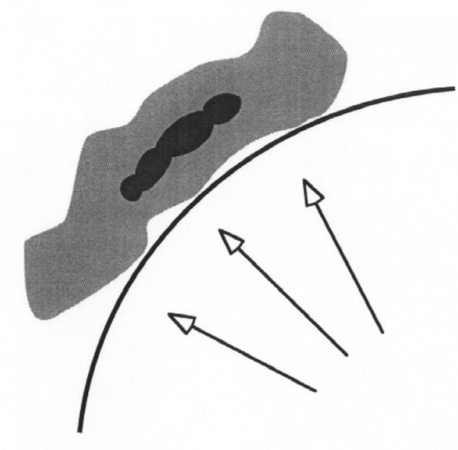

THEN

$\mathrm{HI}$ and CO are co-moving. Bubble (wind) encounters cloud.

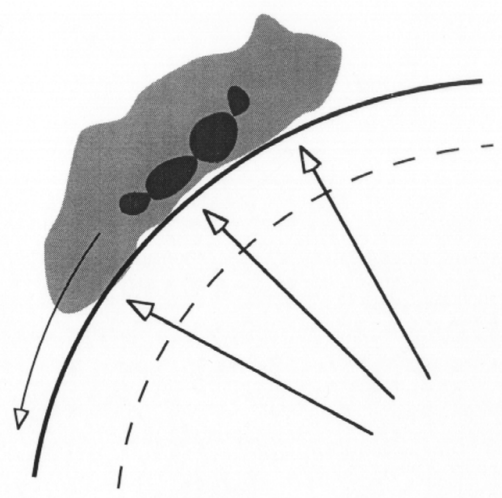

)

FIG. 17.-Cartoon model of the Ursa Major system. At some time in the past (left-hand panel) the $\mathrm{H}$ I and CO in the clouds were moving with the same space velocity. After encountering the wind from the expanding NCP bubble (right-hand panel), an offset in both space and velocity accumulates between the molecular and atomic gas. However, because of the orientation of the clouds, we can observe only the velocity offset. In addition, the clouds are slowly "sliding" down the bubble toward the Galactic plane (curved arrow).

such large IRAS color variations in Ursa Major cannot be attributed to variations in the UV radiation field bathing a cloud but must be related to the small particle population presumed responsible for most of the IRAS 12 and $25 \mu \mathrm{m}$ emission. They found that the recent history of the matter is a determining factor in the abundance of small particles; events that have recently heated dust grains cause the release of small particles from the grain mantles which results in increased 12 and $25 \mu \mathrm{m}$ emission. One way for this to happen is shock heating in a cloud-wind interaction such as our model of Ursa Major.

Heiles, Reach, \& Koo (1988) found that shocks can modify the particle size distribution by either destroying large particles or producing very small particles. They further concluded that very small particles are probably formed in shocks in the $10-20 \mathrm{~km} \mathrm{~s}^{-1}$ range. The expansion velocity of the NCP loop falls neatly in this range, and so it is possible that the particle size distribution at the tips of filaments 1 and 2 has been modified by the interaction with the NCP loop wind. If the NCP loop wind reached these parts of the filaments first, they would have "borne the brunt" of the wind impact and been modified more than regions farther up in the cloud.

\section{CONCLUSIONS}

We have analyzed the morphology and kinematics of the atomic and molecular gas in the Ursa Major molecular clouds. Peculiar features of the clouds include extremely filamentary structure; a tangled $\mathrm{CO}$ velocity field with fitted gradient of about $0.4 \mathrm{~km} \mathrm{~s}^{-1} \mathrm{pc}^{-1}$; a systematic velocity gradient in $\mathrm{H} \mathrm{I}$ of about $0.25 \mathrm{~km} \mathrm{~s}^{-1} \mathrm{pc}^{-1}$; FWHM line width gradient in $\mathrm{H} \mathrm{I}$ of $0.8 \mathrm{~km} \mathrm{~s}^{-1} \mathrm{pc}^{-1}$; offsets of up to 4 $\mathrm{km} \mathrm{s}^{-1}$ between the centroid velocities of the $\mathrm{CO}$ and $\mathrm{H} \mathrm{I}$; and systematic variations of nearly $50 \%$ in IRAS colors. The magnetic field is also significantly larger in the $\mathrm{H} \mathrm{I}$ filament than in the surrounding gas.

We find that these features are consistent with a model in which the clouds lie on the back side of the expanding NCP bubble, either in the upper or lower quadrant. The overall appearance of clouds and the NCP loop, and the upwardpointing arcs seen in $\mathrm{CO}$ and infrared emission, lead us to favor strongly the upper quadrant, even though it requires the assumption of a rotational motion superimposed on the expansion, with the clouds slowly "sliding down" the back side of the bubble. The atomic and molecular gas have been accelerated by the expansion of the bubble, but the atomic gas has been accelerated more so because it is less dense, which gives rise to the velocity difference between the $\mathrm{CO}$ and $\mathrm{H}$ I. An interaction with the wind responsible for the expansion of the NCP bubble can also cause the increase seen in IRAS color ratios at the tips of the largest filaments through shock production of small grains. The line width gradient may be due to an anisotropic velocity dispersion which arises from gas-streaming motions parallel to the filaments.

It is our pleasure to thank Leo Blitz, Eugene deGeus, Edith Falgarone, Carl Heiles, Richard Klein, K. D. Kuntz, Chris McKee, Phil Myers, Ralph Pudritz, Jim Stone, David Schleuning, and Mike Shull for helpful discussions. We thank Rolf Güsten for his assistance in obtaining the Effelsberg $\mathrm{H}$ I data, and Tom Dame and Dap Hartmann for obtaining two wide-band $1.2 \mathrm{~m} \mathrm{CO}$ spectra. Ray Plante was a great help in creating the VRML images of our data. M. W. P. thanks the University of Maryland Astronomy Department for office space and computer use during his 


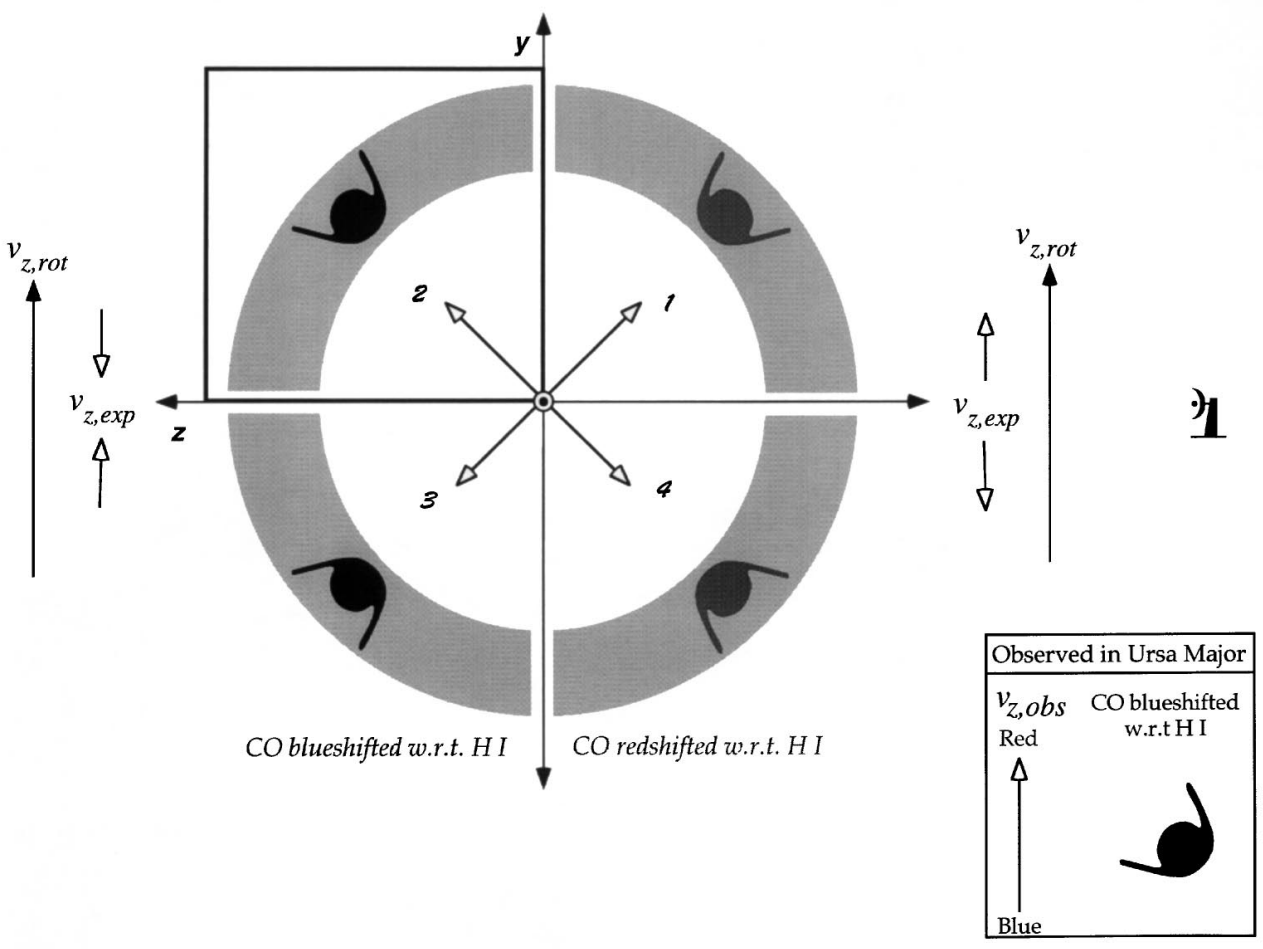

FIG. 18.-Assuming the Ursa Major clouds lie on the exterior of a bubble expanding radially outward, they may be in one of four quadrants (labeled 1, 2, 3 , and 4). For each quadrant, the direction of the observed LSR velocity gradients due to expansion $\left(v_{\exp , z}\right)$ and due to rotation toward the Galactic plane $\left(v_{\text {rot }, z}\right)$ are indicated by arrows. The arrows "point" redward, toward more positive velocities. That the clouds lie in quadrants 1 and 4 may be ruled out because in these quadrants the molecular gas would be redshifted with respect to the atomic gas, whereas we observe it to be blueshifted. The "comets" indicate how objects "blown" by the bubble's wind would appear to an observer. Since we observe such objects to have upward-pointing tails (see, e.g., Fig. 9), we conclude the Ursa Major clouds lie in quadrant 2, on the back side of the NCP bubble.

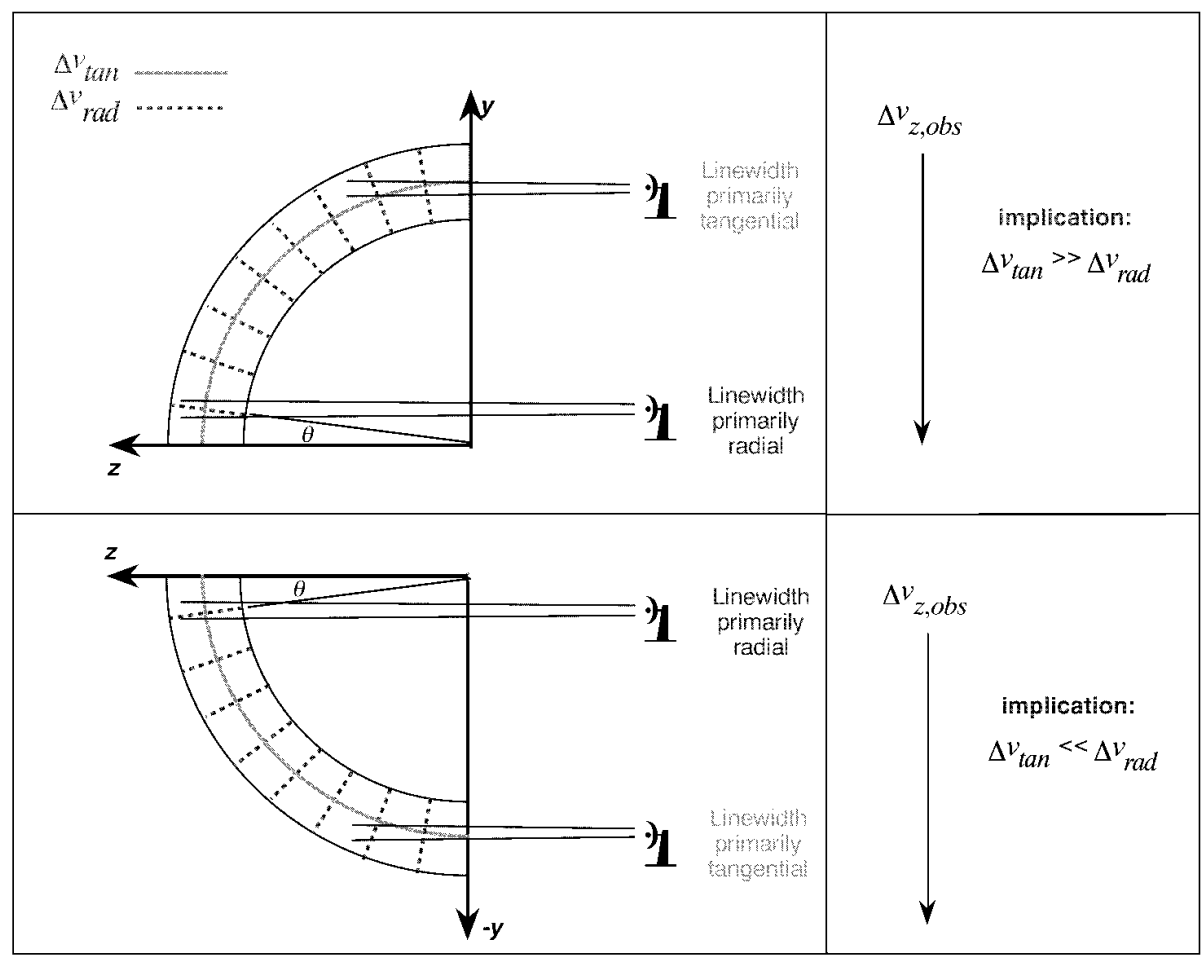

Fig. 19.-One potential explanation for the line width gradient seen in $\mathrm{H}$ I. Assume the three-dimensional velocity dispersion is anisotropic and the line-of-sight component can be decomposed into a "tangential" component $\Delta v_{\tan }$ (solid line) that runs along the length of the filament and a "radial" component $\Delta v_{\text {rad }}$ (dashed line) that runs across it radial to the expanding bubble. Depending on the exact line of sight and which quadrant the filament is in, the measured line width $\Delta v$ will be dominated by $\Delta v_{\text {rad }}$ or $\Delta v_{\text {tan }}$. We observe $\Delta v$ to increase with increasing Galactic latitude. 

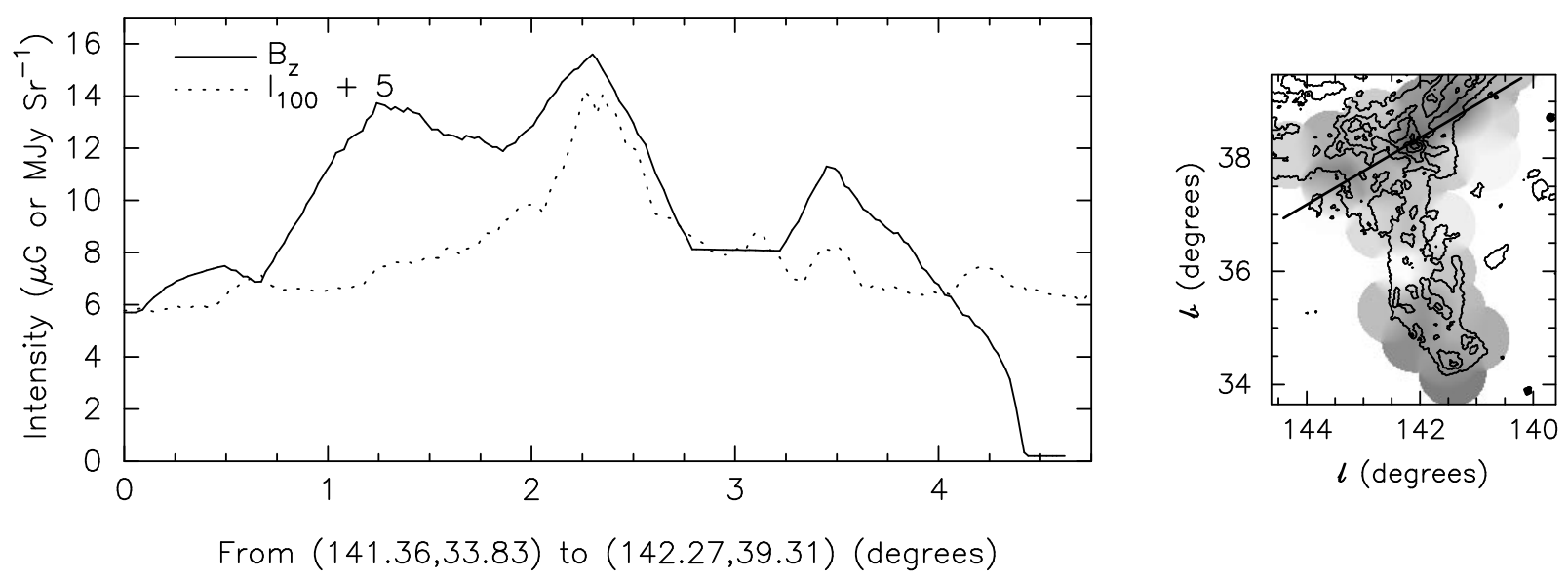

From $(141.36,33.83)$ to $(142.27,39.31)$ (degrees)
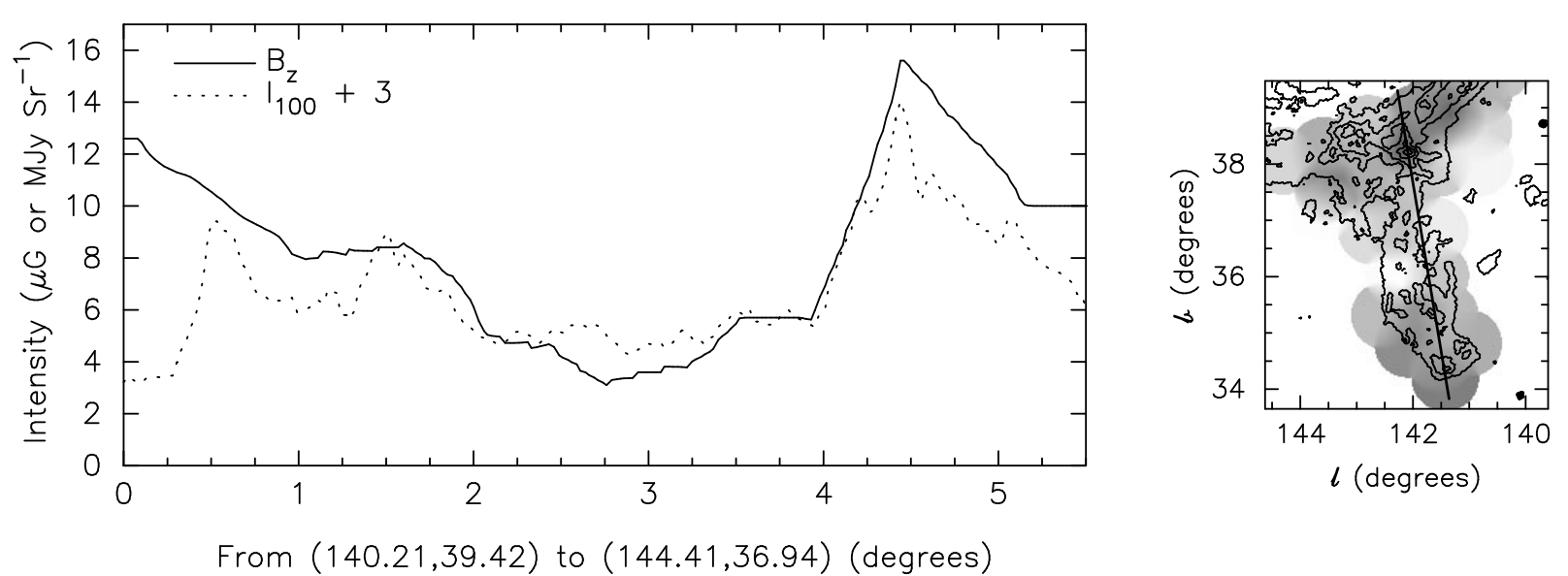

FIG. 20.-Cuts through the H I Zeeman (right-hand panels; gray scale) and IRAS $100 \mu \mathrm{m}$ images (right-hand panels; contours). The positions of the cuts are indicated by the solid lines in the upper and lower right-hand panels. Along the $\mathrm{H} \mathrm{I} / \mathrm{CO}$ filaments (lower left-hand panel), the line-of-sight magnetic field $B_{z}$ and the IRAS $100 \mu \mathrm{m}$ intensity appear extremely well correlated. A cut across the filament (upper left-hand panel) shows $B_{z}$ and $I(100)$ to be uncorrelated except where the cut crosses the filament (at about 2.25 on the $x$-axis). To facilitate comparison, we have added constants to the $I(100)$ values ( $3 \mathrm{MJy} \mathrm{Sr}^{-1}$ in the lower panel and $5 \mathrm{MJy} \mathrm{Sr}^{-1}$ in the upper panel).

visits. We extend our appreciation to Dennis Mumma and Greg Wright for expertly maintaining the Bell Labs $7 \mathrm{~m}$ antenna and its receivers and electronics. This work was supported by National Science Foundation grant NSFFD93-20238.
Blitz, L., Magnani, L., \& Mundy, L. 1984, ApJ, 282, L9

Bloemen, J. B. G. M., et al. 1986, A\&A, 154, 25

Bock, J. J., et al. 1993, ApJ, 410, L115

Boulanger, F., Falgarone, E., Puget, J. L., \& Helou, G. 1990, ApJ, 364, 136

de Vries, H. W., Heithausen, A., \& Thaddeus, P. 1987, ApJ, 319, 72

Elmegreen, B. G. 1987, ApJ, 312, 626

Falgarone, E., \& Perault, M. 1988, A\&A, 205, L1

Ferriere, K. M., Mac Low, M.-M., \& Zweibel, E. G. 1991, ApJ, 375, 239

Gir, B.-Y., Blitz, L., \& Magnani, L. 1994, ApJ, 434, 162

Goodman, A. A., Benson, P. J., Fuller, G. A., \& Myers, P. C. 1993, ApJ, 406, 528

Goodman, A. A., Myers, P. C., Güsten, R., \& Heiles, C. 1994, in ASP Conf.

Proc. 58, The First Symposium on the Infrared Cirrus and Diffuse Interstellar Clouds, ed. R. Cutri \& W. Latter (San Francisco: ASP), 425

Heiles, C. 1984, ApJS, 55, 585

Heiles, C., \& Habing, H. J. 1974, A\&AS, 14, 1

Heiles, C., Reach, W. T., \& Koo B.-C. 1988, ApJ, 332, 313

\section{REFERENCES}

Hildebrand, R. H. 1983, QJRAS, 24, 267

Jahoda, K., Lockman, F. J., \& McCammon, D. 1990, ApJ, 354, 184

Joncas, G., Boulanger, F., \& Dewdney, P. E. 1992, ApJ, 397, 165

Kamaya, H., Mineshige, S., Shibata, K., \& Matsumoto, R. 1996, ApJ, 458, L25

Kuntz, K. D., \& Danly, L. 1996, ApJ, 457, 703

Langer, W. D., Wilson, R. W., \& Beichman C. A. 1989, ApJ, 337, 335

Lockman, F. J., Jahoda, K., \& McCammon, D. 1986, ApJ, 302, 432

Magnani, L., Blitz, L., \& Mundy, L. 1985, ApJ, 295, 402 (MBM)

Meyerdierks, H., Heithausen, A., \& Reif, K. 1991, A\&A, 245, 247

Myers, P. C., Goodman, A. A., Güsten, R., \& Heiles, C. 1995, ApJ, 442, 177

Penprase, B. E. 1993, ApJS, 88, 433

Seki, M. 1990, in Galactic and Intergalactic Magnetic Fields, ed. R. Beck, P. P. Kronberg, \& R. Wielebinski (Dordrecht: Kluwer), 321

Wheelock, S. L., et al. 1991, IRAS Sky Survey Atlas: Explanatory Supplement, VI-27 


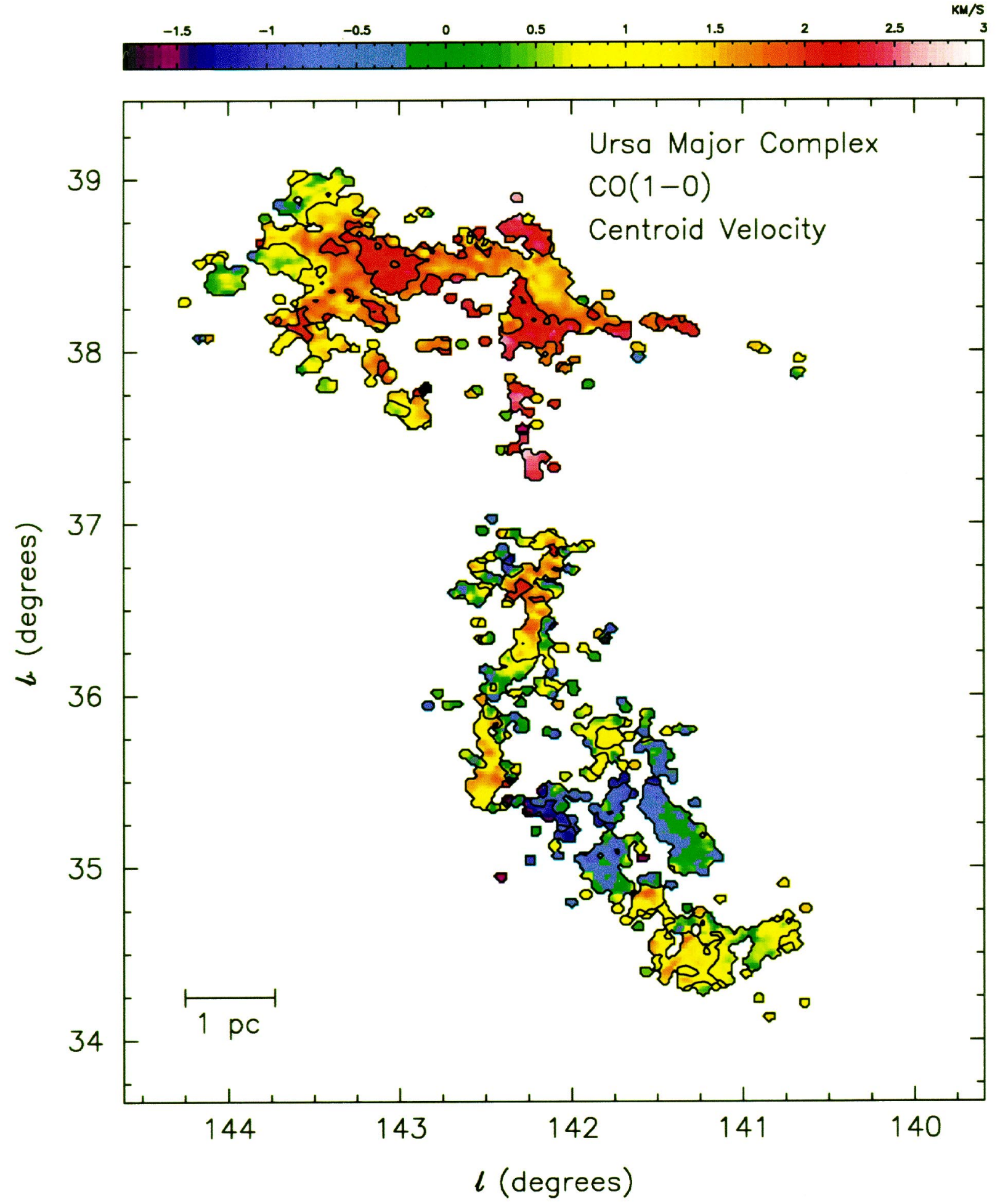

FIG. 10.-Map of centroid LSR velocity of CO. The data have been smoothed to $4^{\prime}$ and clipped where the integrated intensity falls below $3 \sigma$ for the smoothed spectrum. Contours are at $1 \mathrm{~km} \mathrm{~s}^{-1}$ intervals from -2 to $2 \mathrm{~km} \mathrm{~s}^{-1} \mathrm{LSR}$.

Pound \& Goodman (see 482, 339) 


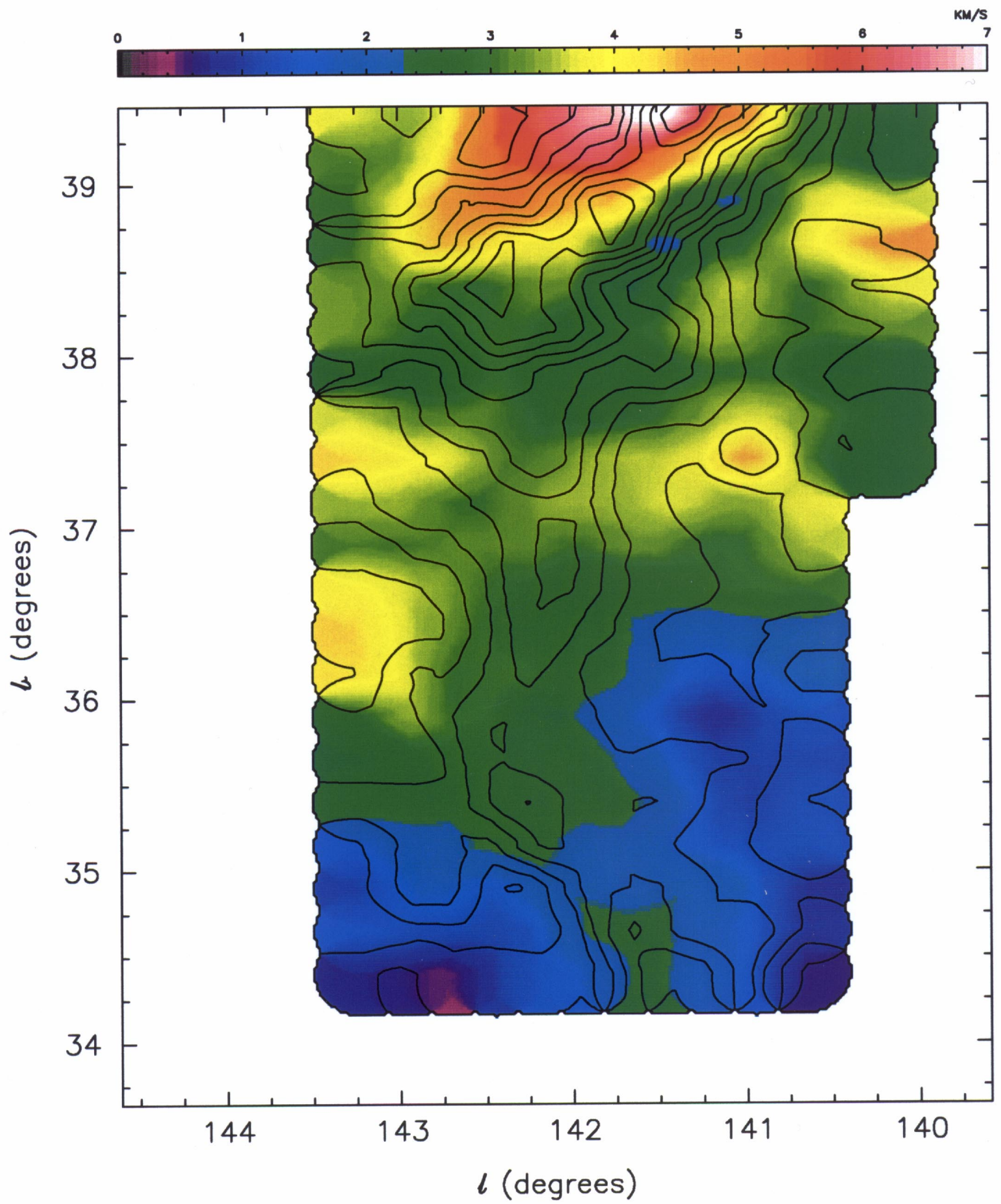

FIG. 13. - Map of centroid LSR velocity of $\mathrm{H} \mathrm{I}$ (color). Contours of peak brightness temperature of $\mathrm{H}$ I are shown at $2.5,5, \ldots, 50 \mathrm{~K}$. These maps show the $\mathrm{H}$ I filament is a distinct kinematic feature. Also, the deviation at $(\ell, b) \sim(142.5,35)$ from the smooth velocity field corresponds to the location of MBM 28 - the CO cloud with the most negative velocity in the complex. Note the range in velocity indicated by the color scale is larger than that in Fig. 10. 